Claremont Colleges

Scholarship@ Claremont

All HMC Faculty Publications and Research

HMC Faculty Scholarship

$1-1-2000$

\title{
Solvent as Electron Donor: Donor/Acceptor Electronic Coupling Is a Dynamical Variable
}

Edward W. Castner Jr.

Rutgers University

Darcy Kennedy '00

Claremont McKenna College

Robert J. Cave

Harvey Mudd College

\section{Recommended Citation}

Castner, E.W. Jr.; Kennedy, D.; Cave, R.J. “Solvent as Electron Donor: Donor/Acceptor Coupling is a Dynamical Variable,” J. Phys. Chem. A 2000, 104, 2869. DOI: 10.1021/jp9936852

This Article is brought to you for free and open access by the HMC Faculty Scholarship at Scholarship @ Claremont. It has been accepted for inclusion in All HMC Faculty Publications and Research by an authorized administrator of Scholarship @ Claremont. For more information, please contact scholarship@cuc.claremont.edu. 


\title{
ARTICLES
}

\section{Solvent as Electron Donor: Donor/Acceptor Electronic Coupling Is a Dynamical Variable}

\author{
Edward W. Castner, Jr.*,† \\ Brookhaven National Laboratory, Chemistry Department, Building 555A, Upton, New York 11973-5000
}

Darcy Kennedy and Robert J. Cave*,:

Harvey Mudd College, Department of Chemistry, Claremont, California 91711

Received: October 14, 1999; In Final Form: December 24, 1999

\begin{abstract}
We combine analysis of measurements by femtosecond optical spectroscopy, computer simulations, and the generalized Mulliken-Hush (GMH) theory in the study of electron-transfer reactions and electron donoracceptor interactions. Our focus is on ultrafast photoinduced electron-transfer reactions from aromatic amine solvent donors to excited-state acceptors. The experimental results from femtosecond dynamical measurements fall into three categories: six coumarin acceptors reductively quenched by $N, N$-dimethylaniline (DMA), eight electron-donating amine solvents reductively quenching coumarin 152 (7-(dimethylamino)-4-(trifluoromethyl)coumarin), and reductive quenching dynamics of two coumarins by DMA as a function of dilution in the nonreactive solvents toluene and chlorobenzene. Applying a combination of molecular dynamics trajectories, semiempirical quantum mechanical calculations (of the relevant adiabatic electronic states), and GMH theory to the C152/DMA photoreaction, we calculate the electron donor/acceptor interaction parameter $H_{\mathrm{DA}}$ at various time frames. $H_{\mathrm{DA}}$ is strongly modulated by both inner-sphere and outer-sphere nuclear dynamics, leading us to conclude that $H_{\mathrm{DA}}$ must be considered as a dynamical variable.
\end{abstract}

\section{Introduction}

Bimolecular electron-transfer reactions in solution frequently have rates limited by diffusion of donor and acceptor molecules, as one or both of the reactant species is usually in a low concentration relative to the solvent. To obtain a detailed mechanistic and kinetic understanding of electron-transfer reactions in solution, chemists have devised ingenious schemes in which the two reactants, the donor and acceptor, are held at a fixed distance and orientation, so that diffusion will not complicate the study of the intrinsic electron-transfer rates. ${ }^{1-3}$ Examples of these studies include tethering the donor and acceptor together via covalent bonds, ${ }^{4-16}$ charge-transfer complexes, ${ }^{17-20}$ peptides, ${ }^{21-23}$ proteins,${ }^{24-31}$ or nucleic acid spacers. ${ }^{32-35}$

In our work, we follow the lead of Yoshihara and coworkers, ${ }^{36-53}$ where we do not fix the orientations of donor and acceptor, but guarantee that no translational diffusion need take place by choosing the solvent to be the electron donor. Because the acceptor will then have its first solvent coordination sphere composed of nothing but donor molecules, an electron-transfer reaction photoinitiated by an ultrashort laser pulse can then provide direct information on the intrinsic reaction rate (and hopefully mechanism) by eliminating the complexities introduced by diffusion. The very rapid rates of electron-transfer observed in a number of systems for which the solvent is the donor imply that the electron is transferred before solvent

† E-mail: castner@ rutchem.rutgers.edu. Present address: Rutgers University, Department of Chemistry, 610 Taylor Road, Piscataway, NJ 08854 8087.

† E-mail: robert_cave@hmc.edu. reorganization is complete and that the rate is faster than reorientational or translational diffusion. Combining methods of femtosecond optical spectroscopy, computer simulations, and the generalized Mulliken-Hush theory ${ }^{54,55}$ for evaluating electron donor/acceptor electronic coupling, we have arrived at a substantially new picture for describing the earliest events in solution phase electron transfer.

Applied sensibly, there is no obvious reason the Marcus theory for bimolecular electron-transfer reactions ${ }^{56}$ should not apply to interpretation of our experimental results. Zusman's extensions of the Marcus theory indicated that the reaction rate could be dominated by a nuclear-factor preexponential term proportional to the inverse solvation time for adiabatic electrontransfer reactions. ${ }^{57-59}$ The Barbara and Yoshihara groups have made use of two-dimensional reaction-coordinate models in analyzing their ultrafast photoinduced electron-transfer rates for intra- and intermolecular reactions, respectively. ${ }^{38,43-47,50,52,60-67}$ They have combined the models of Sumi and Marcus, ${ }^{68}$ and of Bixon and Jortner, ${ }^{69,70}$ so that the electron-transfer reaction proceeds along or between two reaction coordinates: a solvation coordinate, and a vibrational coordinate. An important point is to decide whether the inertial components of solvation dynamics are included along the solvation coordinate or along the vibrational coordinate. The latter is actually sensible, as the inertial dynamics often can be described in terms of specific intermolecular normal modes, such as librations or $\mathrm{H}$-bond vibrations, at least for polar liquids. An excellent review of these theories and relevant ultrafast electron-transfer reactions in solution was published in 1993 by Heitele. ${ }^{71}$

To study this class of ultrafast photoinduced bimolecular 


\section{SCHEME 1}

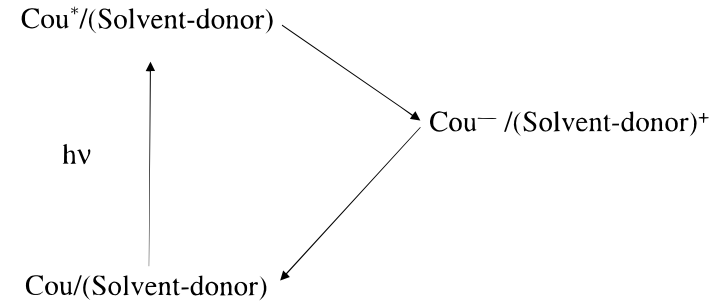

electron-transfer reactions, we have used the excited states of substituted coumarins as electron acceptors, and a variety of electron-rich solvents as donors. The photochemical and photophysical properties of substituted coumarins molecules have been widely studied, because of their broad applications as laser dyes. Jones and co-workers first discovered many of the photoinduced electron-transfer reactions studied here. They demonstrated that the excited singlet states of the coumarins can be both reductively and oxidatively quenched by solvents such as $N, N$-dimethylaniline and nitrobenzene, respectively. ${ }^{72,73}$ Substituted coumarins have been often used as probes of solvation dynamics, where the time-dependent fluorescence Stokes shift (TDFSS) of the coumarin charge-transfer excited state is used as a probe of solvent reorganization dynamics. ${ }^{74-76}$ Reductive quenching of coumarin excited states by electron donors (such as DMA) has been studied as a probe of diffusionlimited reactions at high donor concentrations of $\sim 0.5 \mathrm{M}$, which is at or beyond the so-called "static quenching" concentration limit. ${ }^{77}$ Yoshihara and co-workers have used several classes of organic laser dye molecules to study the ultrafast bimolecular electron-transfer between solvent and excited-state chromophore. ${ }^{37-53}$

In the experimental parts of this paper, we present studies on the photoinduced bimolecular electron-transfer rates from solvent donors to substituted coumarin excited-state acceptors. The experiments may be categorized using three paradigms. In the first group of experiments, we characterized a series of molecules that are likely to be good electron donors by measuring the reductive fluorescence quenching of coumarin 152 in these solvents. In the second series of experiments, we used DMA as the electron donor to reductively quench the excited states of a series of substituted coumarin molecules. The third group of experiments involves the use of DMA to reductively quench the excited state of coumarin 151 or 152 , but the focus is on measuring the bimolecular electron-transfer rate dependence as a function of dilution of the donor solvent with a suitable cosolvent, such as toluene or chlorobenzene.

A diagram of the photophysical and photochemical reactions we have studied is presented in Scheme 1. On excitation by the near-ultraviolet laser pulse $(h v)$, the excited state of the acceptor chromophore (denoted "Cou") is created. Inner-sphere and outer-sphere reorganization dynamics immediately begin to occur; vibrational relaxation and inertial solvent motions predominate at early times. Progress along the electron-transfer reaction coordinate begins simultaneously, and it depends on the free energy driving force and electronic coupling values. Our femtosecond time-resolved emission transients allow us to record wavelength-dependent dynamics that include the reorganization and charge-separation reactions. The geminate charge recombination usually occurs rapidly but is not an observable in our emission experiment.

\section{Experimental and Theoretical Methods}

Experimental Methods. Samples. Coumarins 151 (7-amino4-(trifluoromethyl)coumarin), 152 (7-(dimethylamino)-4-(triflu-

\section{SCHEME 2}
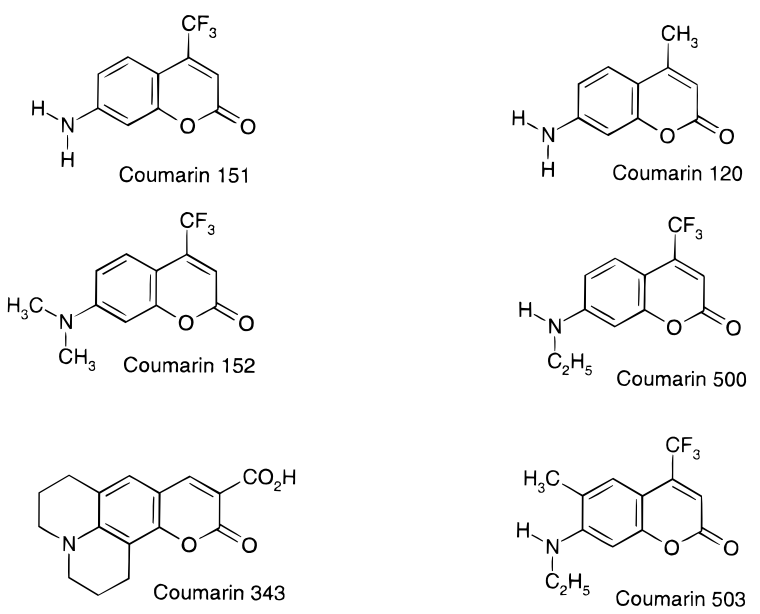

\section{SCHEME 3}
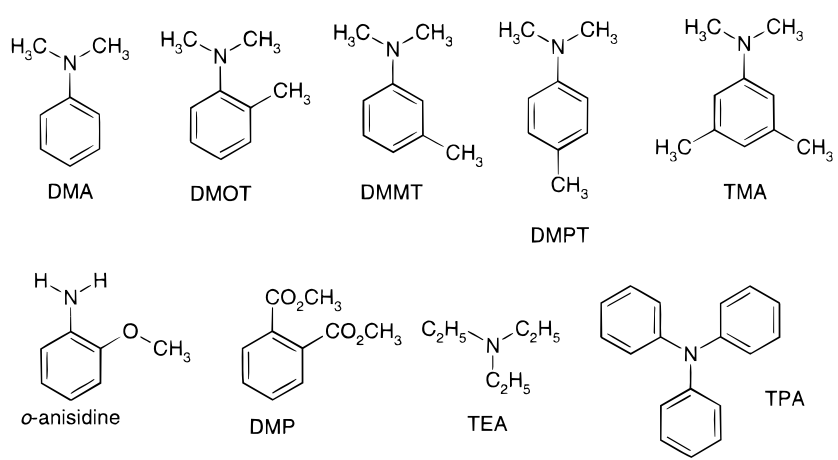

oromethyl)coumarin), 120 (7-amino-4-methylcoumarin), and 343 were Kodak Laser Grade and were used as received. Coumarins 500 (7-(ethylamino)-4-(trifluoromethyl)coumarin) and 503 (aka 307, 7-(ethylamino)-6-methyl-4-(trifluoromethyl)coumarin) were Exciton Laser Grade and were also used as received. Structures of the coumarins are shown in Scheme 2.

Solvent purity grades were ACS reagent, HPLC, or spectrograde, obtained from Aldrich. $N, N$-Dimethylaniline was fractionally distilled at reduced pressure (15 Torr) under an argon atmosphere $\left(\sim 85{ }^{\circ} \mathrm{C}\right)$. Triethylamine (TEA), toluene, chlorobenzene, and benzene were distilled over calcium hydride, under an atmosphere of argon. Triphenylamine (TPA) was obtained from Aldrich (98\%) and used as received. All solvents were transferred by gastight syringes to the sample flow reservoir, which was vigorously purged with argon. $N, N$ Dimethyl-o-, $-m-$, and $-p$-toluidines (DMOT, DMMT, and DMPT, respectively), dimethyl phthalate (DMP), o-anisidine (o-A), and N,N-3,5-tetramethylaniline (TMA) were used as received, but opened in an argon/vacuum/argon-purged glovebag and transferred directly into the argon-purged sample flow reservoir. Scheme 3 shows the structures for the nine electron donors.

Sample Flow System. The sample flow system is designed to flow the solution through a $1 \mathrm{~mm}$ path length fused-silica flow cell (NSG Precision, Type 48H). The principal components of the flow system are the flow cell, the glass sample reservoir with ground-glass top (modified from a 50 or $100 \mathrm{~mL}$ dropping funnel and sealed with a Teflon sleeve), and the pump (magnetically coupled Teflon gear pump, with 316 stainless steel housing, from MicroPump). The components are connected via Teflon tubing, 0.097 in. outer diameter. By using a Teflon sleeve for the ground-glass top, the system can be closed, or purged 
with a slight overpressure of argon gas. All experiments were performed at room temperature, about $292 \mathrm{~K}$.

Fluorescence Upconversion Spectrometer. The femtosecond fluorescence upconversion spectrometer is modeled after the design of Horng et al. ${ }^{74}$ which in turn was developed from the design of Rosenthal et al. ${ }^{78} \mathrm{~A}$ partial description of the instrument has been given previously. ${ }^{79,80}$ The $1 \mathrm{~mm}$ path flowing sample solution is excited at $82 \mathrm{MHz}$ by pulses of about $0.3-0.6 \mathrm{~nJ} /$ pulse energy. These pulses are obtained via second harmonic generation (SHG) in a Type I LBO or BBO angletuned phase-matched nonlinear optical crystal with $0.4 \mathrm{~mm}$ thickness. After the SHG process, the SHG light pulses are reflected from a dichroic beam splitter, then through a fusedsilica retroreflecting prism-pair for independent control of the group-velocity dispersion of the SHG light. TLM1-coated mirrors from CVI Lasers are used for the dichroic and highreflecting mirrors. A quartz zero-order half-wave retarder (Optics for Research) is used to rotate the polarization of the pump pulse in order to select polarization gating of the fluorescence for detecting emission at the magic angle $\left(54.7^{\circ}\right)$ with respect to the excitation polarization. Magic angle fluorescence gating detection was used to eliminate contributions from rotational diffusion to the observed fluorescence decays. The excitation beam was focused tightly into the flowing sample cell with a $10 \mathrm{~cm}$ focal length fused silica lens. The fluorescence was collimated and refocused into the gating crystal by the ellipsoidal reflector (Melles Griot), which has a rhodium reflective coating.

The residual fundamental light after the SHG process passes straight through a dichroic beam splitter and has a computercontrolled delay position obtained by moving a CVI Lasers goldcoated retroreflector on a microstepping translation stage. The translation stage has a time-delay window of $1 \mathrm{~ns}$ and is capable of $0.67 \mathrm{fs} / \mathrm{step}$ closed-loop positioning using a nuStep/nuDrive controller from nuLogic, Inc. and LabVIEW software. ${ }^{81}$ A zeroorder half-wave retarder rotates the polarization of the gate beam from horizontal to vertical, after which a $10 \mathrm{~cm}$ focal length fused silica lens focuses the gate beam onto the Type I angletuned BBO upconversion gating crystal $(0.4 \mathrm{~mm}$ path length). A $5 \mathrm{~mm}^{2}$ gold-coated mirror (Melles Griot) positioned inside the ellipsoidal reflector directs the gate beam into the $\mathrm{BBO}$ upconversion crystal.

The wavelength of the sum-frequency of the fluorescence excited by the SHG pulse and the gating (fundamental) pulse is selected by angle-tuning the BBO upconversion crystal. The fundamental and SHG laser beams are blocked by an iris diaphragm, with the upconverted fluorescence signal or laserpulse third-harmonic generation (THG) cross-correlation signal passing through the iris. These signals are collimated by a 15 $\mathrm{cm}$ focal length fused silica lens. A fused silica prism predisperses the signal to enhance discrimination from scattered second-harmonic light or fluorescence that is not upconverted. About $60 \mathrm{~cm}$ from the prism, a second $15 \mathrm{~cm}$ focal length fused silica lens is used to focus the signal into the $150 \mathrm{~mm}$ spectrometer (Acton SP-150, with a $1200 \mathrm{~g} / \mathrm{mm}$ grating, 300 $\mathrm{nm}$ blaze). A thermoelectrically cooled photomultiplier (Hamamatsu R760-01, selected for low dark counts) is used in singlephoton counting mode with a Stanford SR400 photon counter instrument. A single Schott UG-11 filter is placed at the spectrometer entrance slit to block any scattered laser light. A LabVIEW program controls the data acquisition by reading the intensity of upconverted counts from the SR400 photon counter, then moving the delay stage, to build a histogram of upconverted counts versus time delay. This histogram is the desired upconversion trace and is linearly proportional to the time-dependent fluorescence profile. Usually, several scans are summed to improve the signal-to-noise ratio. For a typical scan, the maximum count rate will be in the range from 100 to 3000 counts/s, with a background of 1-30 counts/s (prior to zerotime delay) caused by scattered laser light. A spectral bandpass of $5.0 \mathrm{~nm}$ is used for the spectrometer.

For the ultrafast fluorescence quenching experiments, the wavelength of observation is chosen to be near the peak of the steady-state emission of the chromophore in a non-electrondonating solvent, usually 475 or $500 \mathrm{~nm}$ (see Supporting Information, Table 7S). Excitation wavelengths ranged from 386.5 to $396 \mathrm{~nm}$. For those solvent-donor/acceptor-chromophore pairs for which the effective electron transfer is fast $\left(>5 \times 10^{11}\right.$ $\mathrm{s}^{-1}$ ), the measured fluorescence dynamics are independent of the wavelength at which the decay is observed. Slower electrontransfer rates mean that the short-time fluorescence decay curve displays a convolution of the diffusive part of the dynamic fluorescence Stokes shift (arising from solvation processes) along with the reductive quenching of the excited state. In these cases, a modest wavelength dependence of the fluorescence dynamics can be observed.

Data Analysis. The instrument temporal response for the femtosecond upconversion experiment is equal to the thirdharmonic cross-correlation between the intensity profiles of the second-harmonic excitation beam and the fundamental gate beam. Typically, the measured third-harmonic cross-correlation fits best to a Gaussian temporal profile, with a full-width, halfmaximum (fwhm) of $160 \mathrm{fs}$, corresponding to $110 \mathrm{fs}$ time resolution, using the standard deconvolution factor of 1.441 for Gaussian pulse correlations. Because of the group-velocity dispersion on the upconverted or third-harmonic light (from the lenses and pre-dispersing prism prior to the spectrometer), the zero-time delay position varies with wavelength. Thus, the zerotime origin is a variable parameter in our data fitting.

Because analysis of fast rise times in the emission transients forms an important part of later sections of the paper, we include our evidence here that the measured THG cross-correlation signal measures the true instrument response exactly, without artifact. To check that this was so, we gated the instantaneous Raman signal of the $\mathrm{C}-\mathrm{H}$ stretch modes of acetone at about $3000 \mathrm{~cm}^{-1}$. The Raman signals of neat acetone were of small amplitude and were noisy but nonetheless were completely superposable upon the THG cross-correlation measured a few minutes later. Other evidence comes from upconversion experiments on coumarin 343 adsorbed on colloidal $\mathrm{TiO}_{2}$. In this case, we observed emission signals that had the rising edge entirely superposable with the rising edge of the THG cross-correlation, with a small deviation on the decaying side indicative of the resolvable excited-state decay. Since the sub-20 fs decay time constants are not surprising for electron injection from a dye into the conduction band of a semiconductor, we believe that this also confirms the fact that the measured THG crosscorrelations are a precise representation of the true instrument temporal response.

The fluorescence decay profiles are fit to sums of exponentials, using standard convolute-and-compare nonlinear least squares techniques. ${ }^{82}$ Specifically, the exponential model function is convolved with the measured THG instrument response function, including a zero-time-delay shift and constant background, until a good quality statistical fit is obtained, as evidenced by the residuals and reduced $\chi^{2}$ value. For short time decays, an exponential rise must always be included in order to obtain the best fit. The model function used is 


$$
K(t)=C+\sum_{i=1}^{n} A_{i} \exp \left[-\left(t-\tau_{\text {shift }}\right) / \tau_{i}\right] \quad n=1-4
$$

where $C$ is a constant and the preexponential factor $A_{i}$ is negative for a rise time. Rise-time amplitudes must equal the sum of the decay amplitudes. For signals with 1000-2500 counts in the peak channel, reduced $\chi^{2}$ values in the range $1.1-1.6$ are obtained coincident with the best fit.

\section{Theoretical Methods}

Molecular Dynamics. Molecular dynamics methods were used to generate a collection of thermally accessible configurations of C152 solvated in DMA. The Cerius2 programs Open Force Field, Minimizer, and Dynamics were used on Brookhaven and Harvey Mudd computers. ${ }^{83}$ The majority of the results presented here come from a single trajectory of $45 \mathrm{ps}$ in duration, but results from two shorter trajectories were used in order to examine particular intramolecular dynamical effects on the electronic coupling. They are described separately below.

Trajectory 1. The Dreiding force field ${ }^{84}$ was used for all atoms, with a torsional restraint applied to the phenyl ringdimethylamino bond on DMA, to yield $\mathrm{Me}-\mathrm{N}-\mathrm{C}_{\text {ring } 1}-\mathrm{C}_{\text {ring2 }}$ torsional angles of approximately $20^{\circ}$. This value is near that obtained by Brouwer and Wilbrandt in their Hartree-Fock and MP2 study of DMA ${ }^{85}$ and is similar to what we obtained using density functional methods. Partial atomic charges were obtained from the charge equilibration method of Rappé and Goddard ${ }^{86}$ for the isolated single molecules and were fixed at the isolated molecule values for the trajectory. The dynamics run employed periodic boundary conditions with 50 DMA and one C152 in a box with dimensions that yield a density of $0.96 \mathrm{~g} / \mathrm{cm}^{3}$ (the density of DMA at $\left.20{ }^{\circ} \mathrm{C}\right) .{ }^{87}$ Ewald sums with a convergence criterion of $2.5 \times 10^{-4} \mathrm{kcal} / \mathrm{mol}$ were used for van der Waals and Coulombic terms. The time step for dynamics was $0.5 \mathrm{fs}$. An initial equilibration of 1 ps was performed using microcanonical ensemble dynamics (NVE) with a $10 \mathrm{~K}$ rescaling window and a target temperature of $300 \mathrm{~K}$, beginning with an energy-minimized structure. This was then followed by $45 \mathrm{ps}$ of canonical ensemble dynamics (NVT) using the Nose-Hoover formalism $^{88}$ with the Verlet leapfrog accelerator. ${ }^{89}$ The NVT dynamics were begun from the last frame of the NVE dynamics, but with randomized velocities. Thus, the target $T$ of $300 \mathrm{~K}$ was not attained until $0.5 \mathrm{ps}$. In addition, there were significant pressure changes in the first ps of NVT dynamics and then a slow pressure decrease (by a factor of 2) over the next 30 ps. There is no discernible correlation between pressure (over the range examined) and relative sizes of $H_{\mathrm{DA}}$ in the results presented below after the initial 5 ps. From this NVT trajectory we selected several frames for analysis.

Trajectory 2. The force field parameters were identical to those used in trajectory 1; however NVE dynamics were performed. The purpose of this trajectory was to follow the motion of the nearest-neighbors of $\mathrm{C} 152$ on a finer time scale, and thus frames were saved every 25 fs. This trajectory was started from the 45 ps snapshot of trajectory 1.

Trajectory 3. In this case we were interested in investigating effects of inversion at the dimethylamino nitrogen on the electronic coupling element. The force field was modified to allow for rotation about the amine-ring bond and to permit inversion of the nitrogen atom. There were small changes of up to $\pm 0.02 \mathrm{e}^{-}$in the equilibrated charges on DMA, but otherwise all other force-field parameters were the same as in trajectory 1 . Once again, the dynamics run employed periodic boundary conditions with 50 DMA and one $\mathrm{C} 152$ in a box with dimensions to yield a density of $0.96 \mathrm{~g} / \mathrm{cm}^{3}$. The same Ewald sums with a convergence criterion of $2.5 \times 10^{-4} \mathrm{kcal} / \mathrm{mol}$ were used for van der Waals and Coulombic terms. The time step for dynamics was $1 \mathrm{fs}$. Specific frames that exhibit inversion of the DMA amine are used from this trajectory; however, the frames come from a portion of the trajectory where the temperature is not fully equilibrated at $300 \mathrm{~K}$. Nevertheless, we expect the qualitative features examined not to be affected by the lack of full equilibration.

Electronic Structure Theory. To evaluate the electronic coupling element between donor and acceptor we used the Generalized Mulliken-Hush (GMH) approach. ${ }^{54,55}$ The GMH method requires adiabatic energies and dipole moments (diagonal and off-diagonal, projected onto the two-state adiabatic dipole moment difference for the pair of adiabatic states that correlate with initial and final diabatic states of interest). ${ }^{54,55}$ The ZINDO semiempirical electronic structure method of Zerner and co-workers ${ }^{90}$ was used to obtain the energies and dipole matrix elements in the present study, with standard parameters (INDO/S; we chose the oxygen $\beta$ value to be the solution phase value of -34.0). We used ZINDO within the Cerius2 computational chemistry environment. ${ }^{83}$ The states of interest for the DMA/C152 pair, as studied experimentally, are the lowest lying singlet excited states of C152 (prepared by laser excitation) and the ground state of the $\mathrm{C}_{152}-/ \mathrm{DMA}^{+}$ion pair. Thus, we required configuration interaction $(\mathrm{CI})$ type wave functions to describe these states.

To determine the subset of DMA molecules within the simulation for which C152/DMA $H_{\mathrm{DA}}$ 's would be calculated, we searched for all DMA that have one or more atoms within $3.5 \AA$ of any atom on the $\mathrm{C} 152$ (reasoning that $H_{\mathrm{DA}}$ decays strongly with distance). For this subset of the DMA within the simulation we then compute $H_{\text {DA }}$ pairwise between each DMA and the $\mathrm{C} 152$. To obtain a consistent definition of the donoracceptor distance $R_{\mathrm{DA}}$, we calculate this from the diabatic difference in dipole moments as $R_{\mathrm{DA}}=\Delta \mu_{\mathrm{d}(12)} / e$, where $\Delta \mu_{\mathrm{d}(12)}$ is the difference in parallel dipole moments for the GMH diabatic states. $\Delta E_{\text {adiabatic }}$, the energy difference between the adiabatic states correlated to the initial and final charge separated states, is defined as $\Delta E_{\text {adiabatic }}=E_{\mathrm{DMA}^{+} / \mathrm{C} 152^{-}}-E_{\mathrm{DMA} / \mathrm{C} 152^{*}}$, where $E_{\mathrm{DMA}^{+} / \mathrm{C}_{152^{-}}}$is the energy of the charge-separated state and $E_{\mathrm{DMA} / \mathrm{C} 152^{*}}$ is the energy of the pre-reactive excited state.

A variety of CI expansions were tested for the C152/DMA pair, allowing excitations from increasingly larger sets of occupied orbitals into all virtual orbitals. It was found that convergence with respect to CI space of the energy and dipole moments was reached for the low-lying excited states when excitations were allowed from the 10 highest lying MOs of the C152/DMA pair. In this case, the CI description of each of the two lowest singlet excited states of $\mathrm{C} 152$ was a mixture of two zeroth-order configurations, one involving the HOMO to LUMO excitation for $\mathrm{C} 152$, the other involving the HOMO-3 to LUMO excitation. This mixing will be a sensitive function of the specific parameters chosen in a semiempirical method, and highlevel calculations have not yet been performed on this system to determine whether this is an accurate description of the lowest excited singlet state. Since we are interested in qualitative trends in the strength of the electronic coupling as a function of position and orientation, we chose to perform a simpler CI calculation, involving excitations out of only the HOMOs on DMA or C152, into all virtual orbitals. In this case, the low-lying singlet state on $\mathrm{C} 152$ is generally dominated by the HOMO-LUMO excitation. The $H_{\mathrm{DA}}$ calculated using this single state is similar 
in magnitude to the averaged electronic coupling element $\left(H_{\mathrm{DA}}{ }^{\text {av }}=\left[\left(H_{\mathrm{DA}(1)}\right)^{2}+\left(H_{\mathrm{DA}(2)}\right)^{2}\right]^{1 / 2}\right)$ obtained using the pair of low-lying multiconfigurational states from the larger CI expansions. In some cases, even when using the small CI expansion, configurations representing the HOMO-LUMO and/or chargetransfer excitations are found to contribute to more than one CI state. In these cases we computed $H_{\mathrm{DA}}$ for the pair of states to which the configurations were the dominant contributors. In some cases this led to unusually short $R_{\mathrm{DA}}$, indicating a multistate (i.e., beyond two-state) treatment should be employed to generate localized diabatic states. However, it is not clear whether these mixings are accurate reflections of the molecular behavior, and since we are interested largely in qualitative trends here, we treat all cases as two-state interactions. We expect that none of the trends we discuss will be significantly altered by this approximation.

We have tested the relative sensitivity of $H_{\mathrm{DA}}$ to the convergence criteria of the $\mathrm{CI}$ energy. In extreme cases, we have observed a shift of about $\pm 5 \mathrm{~cm}^{-1}$ for values of $H_{\mathrm{DA}}$ near $10^{3}$ $\mathrm{cm}^{-1}$ and a worst case of $\pm 3 \mathrm{~cm}^{-1}$ for $H_{\mathrm{DA}}$ values near $1 \mathrm{~cm}^{-1}$. These minor variations do not affect our conclusions.

\section{Experimental Results and Discussion}

Coumarin 152 and Electron-Donating Solvents. Rapid electron transfer from the solvent to the excited state of coumarin 152 was observed from six substituted aniline solvents. A rise time (indicated by a negative preexponential factor $A_{i}$ ) was always required to obtain the best quality fit to the emission data. The fluorescence temporal profiles were highly nonexponential and were fit to sums of two or three decaying exponentials plus a rising exponential. Thus, a simple rate constant for the electron transfer cannot generally be defined. However, following Yoshihara, et al., it is convenient to define the weighted average fluorescence decay time as $\langle\tau\rangle=\Sigma_{i} A_{i} \tau_{i}$ and an effective electron-transfer rate constant $k_{\text {eff }}=1 /\langle\tau\rangle .40,44-46$

DMA reductively quenches the photoexcited $S_{1}$ singlet state of a number of organic chromophores. The assignment of rapid electron transfer for this type of system is made on the basis of ultrafast transient absorption studies carried out by Kandori, et al., on oxazine/dimethylaniline systems ${ }^{39}$ and by Wang, et al., for coumarin $337^{91}$ quenching by DMA. ${ }^{92}$ The radical-cation product $\mathrm{DMA}^{\bullet+}$ has a characteristic absorption feature centered at about $500 \mathrm{~nm}$. The intensity of this spectral feature rises with the same time constant as the excited singlet state and decays via geminate radical recombination on the order of $5 \mathrm{ps}$ for the oxazine $^{\bullet-} / \mathrm{DMA}^{\bullet+}$ pair. The more recent study by Wang et al. conclusively demonstrated the electron transfer to occur from DMA solvent to the excited state of coumarin 337, using ultrafast vibrational spectroscopy. ${ }^{92}$ Our own transient absorption studies of the coumarins in anilines were unsuccessful because the $395 \mathrm{~nm}(3.15 \mathrm{eV})$ excitation allows for a two-photon ionization process of the DMA and the stimulated emission signal from the coumarin excited states is in the same spectral region as the intense $\mathrm{DMA}^{\bullet+}$ solvent absorption. Thus, all of our transients measured at $500 \mathrm{~nm}$ had a nonexponential decay, with a time constant of about $1 \mathrm{ps}$. This preresonance ionization of DMA solvent was not a problem for the Yoshihara group exciting Nile Blue or Oxazine in DMA at > $600 \mathrm{~nm}(2.1 \mathrm{eV}),{ }^{91}$ nor for the Walker group probing in the infrared..$^{92}$

Coumarin 152 (C152) was chosen for use as the excitedstate acceptor chromophore because it has been previously shown ${ }^{40,45,50}$ to have a rapid electron-transfer rate in $N, N$ dimethylaniline (DMA). Comparing coumarin 152/DMA to coumarin 151 (C151)/DMA, we find that use of C152 eliminates any complications that could arise from H-bond donation.

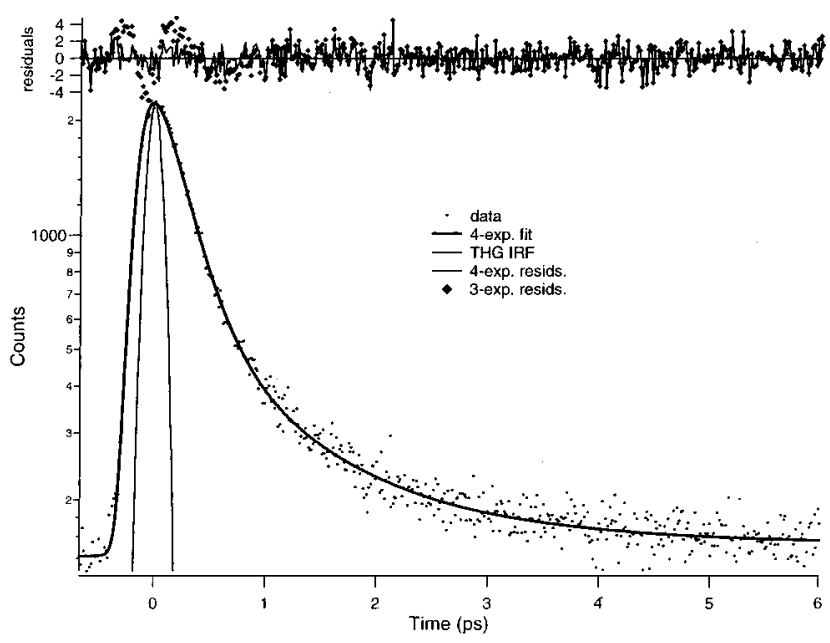

Figure 1. Lower plot: C152 in DMPT fluorescence dynamics from the upconversion data. Note that the decays are nonexponential on all time scales, and a rise time is clearly observable. The best fit to the complete data set is a four-exponential model: one exponential rise with three exponential decay components. The laser THG crosscorrelation signal is shown in the narrow Gaussian centered about zero time delay. Upper plot: residuals from the nonlinear least-squares iterative reconvolution fits. The four-exponential best fit residuals are the solid line; the second-derivative shape for the three-exponential fit is plotted using diamond symbols.

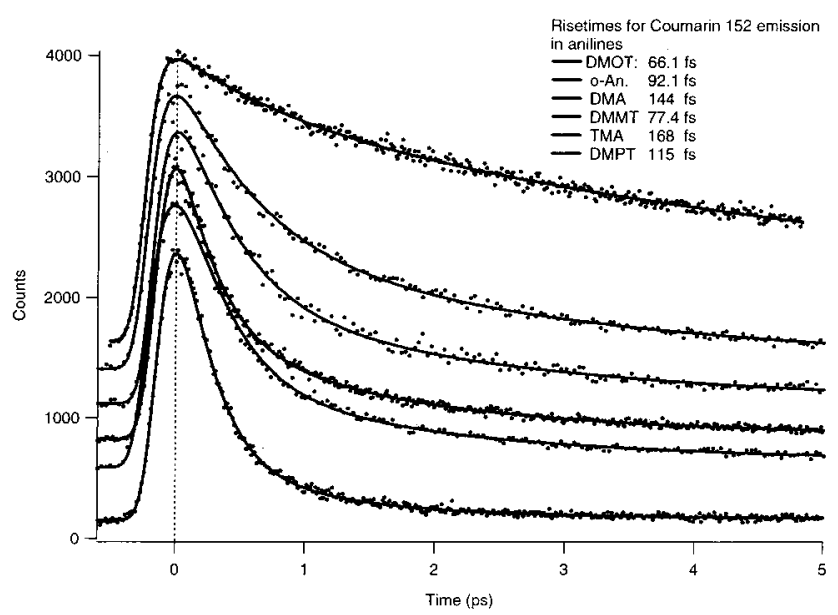

Figure 2. Fluorescence dynamics for coumarin 152 in a series of solvents. From top to bottom, the curves are for C152 in DMOT, $o$-anisidine, DMA, DMMT, TMA, and DMPT solvents.

The fastest electron-transfer rate constant we obtained for an electron-donating solvent to the $\mathrm{C} 152$ excited singlet state was observed for $N, N$-dimethyl- $p$-toluidine (DMPT). As seen in Figure 1, the fluorescence is wholly nonexponential on all time scales. The decay time constants are $220 \mathrm{fs}, 940 \mathrm{fs}$, and $7.1 \mathrm{ps}$, with normalized amplitudes of $91.9,7.7$, and $0.4 \%$, respectively. Perhaps the most significant feature of these data is the rise time of $115 \mathrm{fs}$, without which the data near zero time delay cannot be correctly fit. If the rise time is omitted from the nonlinear least-squares model, a second derivative feature in the residuals indicates the poor quality of the fit, shown in the top of Figure 1. Results of the multiexponential rise/decay fits for reductive quenching of $\mathrm{C} 152$ by the solvent donors are found in Table 1.

Steric Effects of Dimethylaniline Donors. Figure 2 shows the data for rapid fluorescence rise and quenching of coumarin 152 in the dimethylaniline series DMA, DMOT, DMMT, DMPT, and TMA, and also $o$-anisidine. These are derivatives of DMA, with the addition of one or two methyl groups around 
TABLE 1: Emission Decay Amplitudes and Lifetimes, Risetimes, and Effective Electron-Transfer Rate Constants for Coumarin 152 in Electron-Donating Solvents

\begin{tabular}{|c|c|c|c|c|c|c|c|c|c|}
\hline solvent & $A_{1}$ & $\tau_{1}(\mathrm{ps})$ & $A_{2}$ & $\tau_{2}(\mathrm{ps})$ & $A_{3}$ & $\tau_{3}(\mathrm{ps})$ & $\tau_{\text {rise }}(\mathrm{fs})$ & $\tau_{\text {avg }}(\mathrm{ps})$ & $k_{\mathrm{eff}}\left(\mathrm{ps}^{-1}\right)$ \\
\hline DMA & 0.7684 & 0.44 & 0.2316 & 2.49 & & & 144 & 0.917 & 1.09 \\
\hline DMOT & 0.2530 & 1.09 & 0.3735 & 4.22 & 0.3735 & 15.23 & 66.1 & 7.54 & 0.133 \\
\hline DMMT & 0.7749 & 0.29 & 0.2026 & 1.46 & 0.0225 & 7.19 & 77.4 & 0.682 & 1.47 \\
\hline TMA & 0.8767 & 0.29 & 0.0934 & 1.18 & 0.0299 & 4.91 & 168 & 0.513 & 1.95 \\
\hline DMPT & 0.9193 & 0.22 & 0.0768 & 0.94 & 0.0039 & 7.06 & 115 & 0.304 & 3.29 \\
\hline o-anis & 0.6813 & 0.65 & 0.3187 & 3.30 & & & 92.1 & 1.49 & 0.670 \\
\hline TEA & 0.4310 & 28.0 & 0.2663 & 78.8 & 0.3027 & 196.6 & & 92.6 & $1.08 \times 10^{-2}$ \\
\hline TPA/Bz & 0.3767 & 33.9 & 0.5925 & 150.0 & 0.0308 & 405.7 & & 114.0 & $8.78 \times 10^{-3}$ \\
\hline DMP & 0.3138 & 67.8 & 0.4566 & 704 & 0.2296 & 5000 & & 1490 & $6.71 \times 10^{-4}$ \\
\hline
\end{tabular}

The rise time amplitude is the negative of the sum of the decay amplitudes.

the benzene ring. The electron-transfer rate constants vary by a factor of 20 through this range of solvent donors.

We must consider the structural changes at the nitrogen atom between the ground state and radical-cation state in this series of solvents to understand the anomalously slow rate of DMOT versus the rapid DMPT rate. The nitrogen atom in aniline and $N, N$-dimethylaniline is known to have a tetrahedral geometry ( $\mathrm{sp}^{3}$ hybridization) in the neutral ground electronic state of these molecules. On excitation to the first electronic singlet state, the hybridization of this nitrogen atom changes to $\mathrm{sp}^{2}$, leading to a planar geometry for the molecules. Both experiment and molecular orbital calculations have shown that the ground-state radical cation assumes a planar geometry in aniline and $N, N$ dimethylaniline, ${ }^{85}$ which is very similar to the excited-state geometry. By examining molecular models of DMOT, it is easy to see that the ortho methyl group steric hindrance with the $\mathrm{N}, \mathrm{N}$-dimethylamino group prevents a planar conformation from being achieved. The fact that the radical cation cannot achieve the stable planar form in the ortho case will change the oxidation potential relative to the para case, for which no steric hindrance exists. This change in the driving force for the electron-transfer reaction explains the factor of 20 difference in rate between the $N, N$-dimethylamino ortho and para toluidines.

Table 1 also includes results for other types of electron-rich solvents that have been tested. $o$-Anisidine is a good electrondonating solvent, which has a rapid photoreaction rate with C152. ${ }^{52}$ Dimethyl phthalate (DMP) was tested as an electron donor. Because the lifetime is only a factor of 4-10 less than a typical excited-state lifetime of the $\mathrm{C} 152$ acceptor, we must question whether any electron transfer occurs for DMP. Because triphenylamine (TPA) is a solid with a melting point of 122 ${ }^{\circ} \mathrm{C}$, we were not able to measure the electron-transfer rate in a solution of TPA. However, the photoreaction rate for a saturated solution with $13.0 \%$ mole fraction of TPA in benzene was measured. The effective rate constant for electron transfer of $\sim 9 \times 10^{9} \mathrm{~s}^{-1}$ is slow enough to allow for completion of the dynamical solvation processes, as well as for substantial orientational and translational diffusion prior to the reaction. This is despite the fact that the statistical picture of the composition of the solution would lead us to believe that at least one or two TPA electron-donor molecules should be in contact with the C152 acceptor. However, as we discuss at length in the theory section, mere contact between donor and acceptor does not necessarily mean that the electronic coupling will move into the adiabatic regime.

Triethylamine (TEA) donates an electron to the excited state of $\mathrm{C} 152$ with a much slower rate constant than for any of the other substituted anilines. The oxidation potential for TEA is $+0.96 \mathrm{~V}$ (vs $\mathrm{SCE}$ in $\mathrm{CH}_{3} \mathrm{CN}$ ), ${ }^{93}$ a value slightly larger than for aniline $\left(+0.93 \mathrm{~V}\right.$ vs $\mathrm{SCE}$ in $\left.\mathrm{CH}_{3} \mathrm{CN}\right)$ and substantially higher than for DMA $\left(+0.756 \mathrm{~V}\right.$ vs $\mathrm{SCE}$ in $\left.\mathrm{CH}_{3} \mathrm{CN}\right)$. On the basis of driving force arguments, one might expect that TEA could donate an electron about as rapidly as aniline (C152/aniline has an effective rate constant of $\left.1.4 \times 10^{11} \mathrm{~s}^{-1}\right) .{ }^{50}$ Of course, we must remember that the almost 3-fold larger dielectric constant for aniline versus TEA means that the aniline driving force will be greater when ion-pair stabilization effects are also considered. However, our observed rate constant for TEA of $1.1 \times 10^{10}$ $\mathrm{s}^{-1}$ is more than 1 order of magnitude slower. Since the known (relative) free energies for the electron-transfer reaction do not explain the observed rates, we must assume that there is a substantially greater electronic coupling for the aromatic amines than for aliphatic TEA.

Indirect evidence for a smaller electronic coupling for TEA than for the substituted anilines is obtained by analogy to the recent observations of Shirota et al. For a series of three hydrazine solvents acting as electron donors to five coumarin excited states, they observed rate constants slower than would be predicted by reaction free energy parameters alone. ${ }^{51}$ Though the oxidation potential of $\mathrm{N}, \mathrm{N}$-dimethylhydrazine $(+0.346 \mathrm{~V})$ is substantially less than either phenylhydrazine or $N$-methyl$N$-phenylhydrazine $(+0.399$ and $+0.421 \mathrm{~V}$, respectively, all values measured vs $\mathrm{SCE}$ in $\mathrm{CH}_{3} \mathrm{CN}$ ), the electron-transfer rate constants are smallest for the $N, N$-dimethylhydrazine. The observed rates slow by a factor of $30-45$ on going from the phenylhydrazines to dimethylhydrazine. ${ }^{51}$ The proposed explanation is that the electronic coupling for dimethylhydrazine is much less than for the phenylhydrazines. Since electronic coupling is fundamentally related to the donor and acceptor orbital overlap, it is possible that the electronic coupling for TEA versus anilines and TPA is analogous to the case of dimethylhydrazine compared with the two phenylhydrazines.

Dimethylaniline as Electron Donor to Excited States of a Series of Six Coumarins. $N, N$-Dimethylaniline reductively quenches the $S_{1}$ state of many organic chromophores, either when acting as a bimolecular quencher ${ }^{72,73}$ or solvent ${ }^{37-53}$ or when tethered directly to the chromophore. ${ }^{63,94} \mathrm{We}$ obtain photoinduced electron-transfer rates from DMA to a series of six coumarin excited states via ultrafast fluorescence dynamics observed in the upconversion experiment. Figure 3 summarizes these data sets, showing how the ideal fit is obtained only after including the rise time parameter in the fit. The results of multiexponential fitting to these fluorescence dynamics data are given in Table 2, listed in order of increasing electron-transfer rate. Both the shortest and intermediate time constants decrease ( $\tau_{1}$ and $\tau_{2}$, respectively) through this series of coumarins effective electron-transfer rate constant. However, though the amplitudes of $\tau_{1}$ always dominate, the ratio of amplitudes $A_{1} /$ $A_{2}$ increases as we descend through Table 2 from C343/DMA to C151/DMA, with increasing electron-transfer rate constants.

The Yoshihara group has well characterized a large number of these systems, including two reported on here: coumarins 


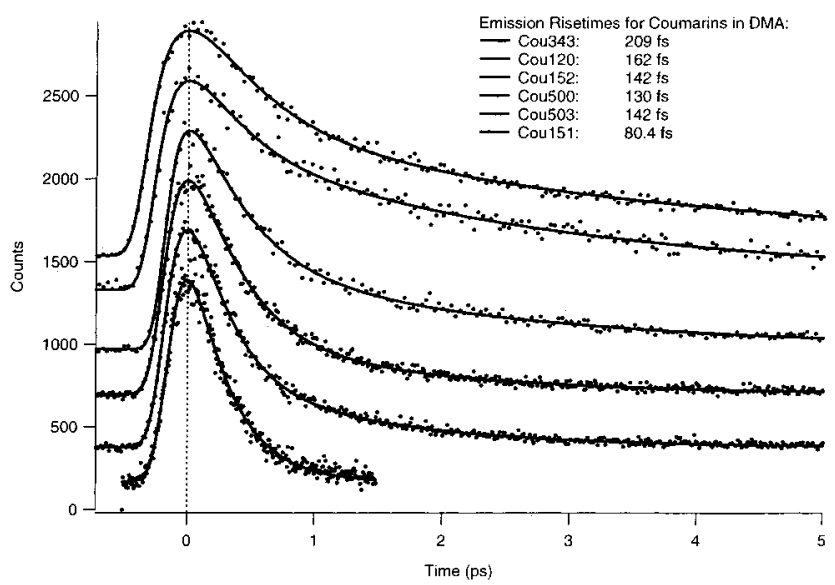

Figure 3. Fluorescence dynamics for a series of six coumarins in dimethylaniline solvent, in order of increasing electron-transfer rate. From top to bottom, the curves are for coumarins $343,120,152,500$, 503 , and 151

151 and $152 .{ }^{40,42,43,45,50}$ To within experimental error, we obtain quantitative agreement with the rates published previously. We report on four DMA/coumarin pairs not previously studied: coumarins 120, 343, 500, and 503. Qualitatively, we agree strongly with the picture of electron transfer with solvent as donor put forward by the Yoshihara group, ${ }^{37-53}$ as well as more recently by Walker et al..$^{92}$ and the Zinth group. ${ }^{95,96}$

The Yoshihara group studied a series of substituted coumarins, where the substituent at the 4-position was chosen from $\mathrm{R}=\left\{\mathrm{H}, \mathrm{CH}_{3}, \mathrm{CF}_{3}\right\}$, and the 7-amino functional group $\mathrm{NX}_{2}$, with $\mathrm{X}$ chosen from $\mathrm{H}, \mathrm{CH}_{3}, \mathrm{C}_{2} \mathrm{H}_{5}$, fused ring, and julolidyl double-ring. ${ }^{40,42,43,45,50}$ They found a trend in the series for which the rates increased with 4-position functionality in the order $k_{(\mathrm{CH} 3)}<k_{(\mathrm{H})}<k_{\left(\mathrm{CF}_{3}\right)}$, and for the 7-position $k_{\text {(double-ring) }}<$ $k_{\text {(single-ring) }}<k_{\left(\mathrm{C}_{2} \mathrm{H}_{5}\right)}<k_{\left(\mathrm{CH}_{3}\right)}<k_{(\mathrm{H})}$.

While it seems plausible that low-frequency torsional modes of the 7-amino group could be participating in a twisted intramolecular charge-transfer (TICT) mechanism, this does not explain our data for the substantially larger rate for C500 and $\mathrm{C} 503$ in DMA relative to $\mathrm{C} 152 / \mathrm{DMA}$. The $\mathrm{NHC}_{2} \mathrm{H}_{5}$ functionality has a very similar moment of inertia, as well as frictional drag on the amino twist, compared to the $\mathrm{N}\left(\mathrm{CH}_{3}\right)_{2}$ group. However, there appears to be an effect due to an amino proton interaction with the solvent that enhances the rate.

To within experimental error, the rates for the C500/DMA and C503/DMA photoredox couples are the same. Since the molecular structures of C500 and C503 are identical save for addition of the 6-methyl group to $\mathrm{C} 503$, we assume that the electronic coupling between either of these two coumarins with DMA will vary only slightly. If this is so, this indicates that the addition of the 6-methyl group to the coumarin does not significantly change the reduction potential, since the electrontransfer rate does not change.

The difference between $\mathrm{C} 120$ and $\mathrm{C} 151$ is that the 4-positions are substituted with methyl versus trifluoromethyl functional groups, respectively. Consistent with the trend observed by Yoshihara et al., ${ }^{40,42,43,45,50} k_{\text {eff, the effective rate constant for }}$ photoinduced electron transfer, is 6.8 times slower for $\mathrm{C} 120$ than for $\mathrm{C} 151$. Clearly, the $\mathrm{CF}_{3}$ electron-withdrawing group on C151 makes the reduction more favorable.

Though C343 has the 7-amino group in a torsionally locked position (because of the double fused (julolidyl) ring system), the electron-transfer rate is still relatively fast. This indicates that restriction of the 7-amino twist does not necessarily slow the electron transfer, despite the slow rate of photoinduced electron transfer measured by Yoshihara et al. for coumarin 153/ DMA, $k_{\text {eff }}=0.054 \mathrm{ps}^{-1} \cdot{ }^{40,42,43,45,50} \mathrm{C} 153$ has a rate-enhancing 4-trifluoromethyl group, does not have a 3-carboxylic acid group, but has the same 7-amino julolidyl double-ring structure as C343. The $k_{\text {eff }}$ for C343/DMA is $0.582 \mathrm{ps}^{-1}, 1$ order of magnitude greater than that for C153/DMA.

The most significant finding for these ultrafast data sets is that for each of the six coumarin/DMA emission profiles, an ultrafast rising component of the transient exists that is consistent with a dynamic Stokes shift arising from inertial solvent dynamics. For each data set, a rise time was required to obtain a proper fit. The value of the rise time varied from a minimum of 80 to a maximum of $209 \mathrm{fs}$, with an average value of $144 \mathrm{fs}$. The rise times should not be identical for each of the six coumarins because we measured the fluorescence at a constant observation wavelength of $500 \mathrm{~nm}$. However, the steady-state emission peaks and spectral widths are all different for each of the six coumarins. This means that we sample slightly different parts of the dynamic fluorescence Stokes shift for each coumarin. For the other six solvent molecules for which rapid electron transfer from solvent to the excited state of coumarin 152 was observed, a rise-time was also required to obtain the best quality fit to the emission data. Only for coumarin 151 in DMA solvent was a single-exponential adequate to describe the decays. Shirota et al. found that, by fitting a higher quality data set for C151/DMA, they obtain a slightly biexponential response, with $A_{1}=0.96, \tau_{1}=200 \mathrm{fs} ; A_{2}=0.04, \tau_{1}=600 \mathrm{fs} .{ }^{50}$ All of the other fluorescence decay profiles were highly nonexponential and were fit to sums of two or three decaying exponentials.

Yoshihara and co-workers have extensively studied many aspects of the solvent-as-donor problem. They have paid special attention to time-dependent fluorescence Stokes shift studies of the solvent reorganization dynamics. ${ }^{45,46}$ Their data show that more than $90 \%$ of the solvent reorganization occurs with two long biexponential time constants, averaging to about $15 \mathrm{ps}$ for DMA. However, another study of solvent reorganization in pure liquid DMA (and other anilines) was also undertaken by this group. Using femtosecond Kerr studies, they found that like all other aromatic liquids, an underdamped librational band is observed for DMA (peak frequency at about $65 \mathrm{~cm}^{-1}$ ). ${ }^{49}$ Certainly intermolecular phenyl librational motions will contribute to this band, and torsional motions of the dimethylamino group will also occur in the $20-80 \mathrm{~cm}^{-1}$ range. Despite the presence of underdamped modes with a librational period of

TABLE 2: Emission Decay Amplitudes, Time Constants, and Effective Electron-Transfer Rate Constants for Coumarins in $N, N$-Dimethylaniline

\begin{tabular}{cccccccccc}
\hline coumarin & $A_{1}$ & $\tau_{1}(\mathrm{ps})$ & $A_{2}$ & $\tau_{2}(\mathrm{ps})$ & $A_{3}$ & $\tau_{3}(\mathrm{ps})$ & $\tau_{\text {rise }}(\mathrm{fs})$ & $\tau_{\text {avg }}(\mathrm{ps})$ & $k_{\text {eff }}\left(\mathrm{ps}{ }^{-1}\right)$ \\
\hline C343 & 0.7110 & 0.485 & 0.2435 & 3.09 & 0.0455 & 13.7 & 209 & 1.719 \\
C120 & 0.6584 & 0.369 & 0.2936 & 2.59 & 0.0480 & 13.5 & 162 & 1.650 \\
C152 & 0.7684 & 0.443 & 0.2316 & 2.49 & & & 142 & 0.582 \\
C500 & 0.8706 & 0.373 & 0.1294 & 1.65 & & & 130 & 0.606 \\
C503 & 0.8922 & 0.251 & 0.1078 & 1.25 & & & 142 & 0.5382 \\
C151 & 1 & 0.309 & & & & & 80.4 & 0.3586 & 1.86 \\
\end{tabular}

The rise time amplitude is the negative of the sum of the decay amplitudes. 


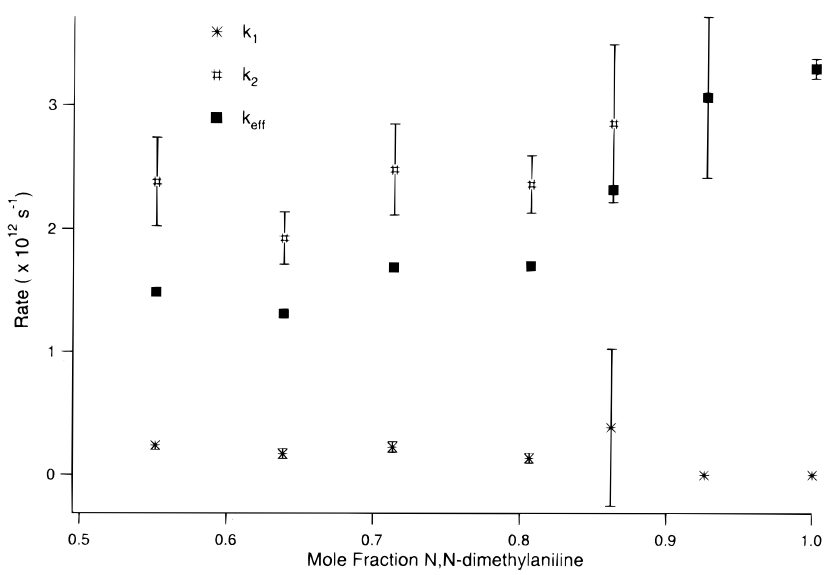

Figure 4. Electron-transfer rate constants for coumarin 151 in DMA/ toluene solvent, as a function of mole fraction DMA. Data are given in Table 3.

TABLE 3: Emission Decay Amplitudes and Lifetimes, and Effective Electron-Transfer Rate Constants for C151/DMA, versus the Mole Fraction of DMA in Toluene

\begin{tabular}{cccccc}
\hline$X_{\text {DMA }}$ & $A_{1}$ & $\tau_{1}(\mathrm{ps})$ & $A_{2}$ & $\tau_{2}(\mathrm{ps})$ & $k_{\text {eff }}\left(\mathrm{ps}^{-1}\right)$ \\
\hline 1.000 & 1.000 & 0.30 & 0.000 & & 3.30 \\
0.926 & 1.000 & 0.33 & 0.000 & & 3.07 \\
0.862 & 0.783 & 0.35 & 0.217 & 2.58 & 1.20 \\
0.806 & 0.702 & 0.42 & 0.298 & 7.35 & 0.402 \\
0.713 & 0.649 & 0.40 & 0.351 & 4.46 & 0.547 \\
0.638 & 0.650 & 0.51 & 0.350 & 5.92 & 0.415 \\
0.551 & 0.582 & 0.42 & 0.418 & 4.20 & 0.500
\end{tabular}

about 300 fs, these motions do not seem to contribute a large fraction to the overall energy relaxation of the time-resolved Stokes shift. However, our excited-state fluorescence dynamics show a rise time that can be assigned to the inertial solvent motions.

To summarize our collection of DMA reductive quenching data for the series of coumarins:

1. The rise times of emission, excited on the red edge of the coumarin absorption spectrum and observed on the red side of the emission spectrum are consistent with inertial solvent reorganization dynamics occurring before the rapid electrontransfer events.

2. Large changes in electronic coupling may require only a small amplitude of solvent libration (vide infra, Theoretical Results and Discussion). There is a coincidence in our average rise times observed for fluorescence dynamics of coumarins in DMA, and one-half of the librational period as obtained from the Kerr data of Smith et al. ${ }^{49}$

Rates of Photoinduced Electron-Transfer from DMA to Coumarin as a Function of Dilution with a Cosolvent. Two studies of the photoinduced bimolecular electron-transfer rate have been carried out as a function of the donor concentration, [DMA]. The first experiment measures the photoinduced electron-transfer rate from DMA to $\mathrm{C} 151$, as a function of dilution with toluene cosolvent. The second study measures the rate of electron transfer from DMA to $\mathrm{C} 152$ as a function of dilution with chlorobenzene.

The photoinduced bimolecular electron-transfer from DMA to $\mathrm{C} 151$ was measured over the DMA mole fraction range from $X_{\mathrm{DMA}}=1.0$ down to 0.55 . The $\mathrm{C} 151$ fluorescence dynamics as a function of dilution of DMA by toluene are shown in Figure 4. The electron-transfer rates and amplitudes for the toluene/ DMA solvent mixtures (from the multiexponential fits to the fluorescence data) are collected in Table 3. The singleexponential character of the decay persists for dilutions of $X_{\mathrm{DMA}}$

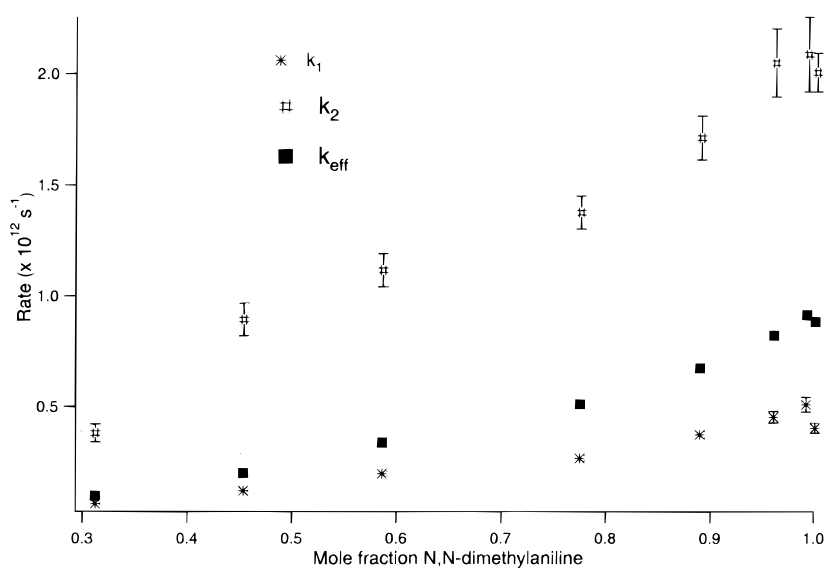

Figure 5. Electron-transfer rate constants for coumarin 152 in DMA/ chlorobenzene solvent, as a function of mole fraction DMA. Data are given in Table 5.

TABLE 4: Properties of $N, N$-Dimethylaniline (DMA) and Chlorobenzene

$\begin{array}{lll}\text { dipole moment }(\mathrm{D}) & 1.61 & 1.62 \\ \text { dielectric constant }\left(\epsilon_{0}\right) & 5.01 & 5.62 \\ \text { viscosity }(\mathrm{cP}) & 1.29 & 0.80 \\ \text { volume }(\mathrm{vdW}, \AA) & 120.6 & 97.1\end{array}$

from 1.0 to 0.925 . For dilutions beyond $X_{\mathrm{DMA}}=0.86$ or lower, the exponential fluorescence decay becomes biexponential. The shorter electron-transfer time constant $\tau_{1}$, similar to the $X_{\mathrm{DMA}}=1.0$ rate, drops dramatically in amplitude. Upon further dilution, the shorter lifetimes increase only slightly, but the longer lifetimes increase sharply. Similar rise times are observed for the C151 in toluene/DMA mixed solvent compared to the C151/DMA emission transients.

Assuming that $\mathrm{C} 151$ has 15 near-neighbor solvent molecules in van der Waals contact, for $X_{\mathrm{DMA}}=0.862$ we would find 13 DMA molecules as near neighbors, if solvent molecules are distributed statistically. On going from pure DMA solvent to $86.2 \%$ mole fraction, a dramatic decrease in the effective electron-transfer rate constant occurs, by a factor of 2.75. Since the free energy driving force for electron transfer is nearly invariant for changes in $X_{\mathrm{DMA}}$, the decrease in electron-transfer rate with decreasing $X_{\mathrm{DMA}}$ must be explained by a change in electronic coupling, $H_{\mathrm{DA}}$. A change in cosolvent mole fraction will not affect $H_{\mathrm{DA}}$ if considered pairwise, but it is possible that a special DMA location with a large, adiabatic $H_{\mathrm{DA}}$ is blocked by a toluene solvent. In this case, rapid adiabatic electron transfer could be prevented, since one or more toluene molecules are (statistically) present in the first solvent layer.

Toluene and DMA have different sizes, dielectric constants, and dipole moments. Because of this, we sought for our next study to find an aromatic solvent that has dipole and dielectric parameters that match DMA. Chlorobenzene was chosen for the second study in order to have an aromatic cosolvent with nearly identical dipolar, quadrupolar, and static dielectric properties when compared to DMA, ${ }^{97}$ as illustrated by the parameters listed in Table 4 . We measured the electron-transfer rates from DMA to the coumarin 152 excited state, as a function of mole fraction of added chlorobenzene.

The experimental data for electron-transfer rates from DMA to coumarin 152, given as a function of the mole fraction of chlorobenzene diluent, are plotted in Figure 5 and listed in Table 5. As $X_{\mathrm{DMA}}$ is decreased from unity to 0.312 , the effective electron-transfer rates decrease by nearly 1 order of magnitude, from 0.88 to $0.097 \mathrm{ps}^{-1}$. Over this concentration range, if the 
TABLE 5: Emission Decay Amplitudes and Lifetimes, and Effective Electron-Transfer Rate Constants for C152/DMA, versus the Mole Fraction of DMA in Chlorobenzene

\begin{tabular}{cccccc}
\hline$X_{\text {DMA }}$ & $A_{1}$ & $\tau_{1}(\mathrm{ps})$ & $A_{2}$ & $\tau_{2}(\mathrm{ps})$ & $k_{\mathrm{eff}}\left(\mathrm{ps}^{-1}\right)$ \\
\hline 1.000 & 0.681 & 0.49 & 0.319 & 2.48 & 0.883 \\
0.992 & 0.587 & 0.48 & 0.413 & 1.97 & 0.915 \\
0.960 & 0.577 & 0.49 & 0.423 & 2.21 & 0.822 \\
0.889 & 0.570 & 0.58 & 0.430 & 2.68 & 0.674 \\
0.775 & 0.592 & 0.73 & 0.408 & 3.75 & 0.510 \\
0.586 & 0.503 & 0.90 & 0.497 & 5.05 & 0.337 \\
0.454 & 0.461 & 1.12 & 0.539 & 8.33 & 0.200 \\
0.312 & 0.439 & 2.63 & 0.561 & 16.3 & 0.097
\end{tabular}

near-neighbor solvent molecules surrounding the coumarin 152 are statistically averaged, we would expect to see the number of DMA solvent/donor molecules decrease from $15\left(x_{\mathrm{DMA}}=\right.$ $1.0)$ to less than $5\left(x_{\mathrm{DMA}}=0.312\right)$. Relative to C152/DMA, similar rise times are observed for the emission transients measured for $\mathrm{C} 152$ in binary chlorobenzene/DMA solvent.

Though the data in Figures 4 and 5 must be interpreted with care, we propose a description of the early time events in photoinduced electron transfer with the following key elements:

i. Statistical averaging occurs between the chlorobenzene and DMA solvent molecules surrounding the $\mathrm{C} 152$ acceptor solute. Since the dipole moment and dielectric constants are the same to within $5 \%$ and the shapes are similar, we can assume that the pairwise electrostatic interactions will be similar.

ii. The DMA molecules in contact with coumarin 152 have very different probabilities for donating an electron. Some are much more likely to become electron donors at a given instant than others. The highest occupied molecular orbitals (HOMOs) of both electron acceptor (C152) and donor (DMA) are highly anisotropic, so the relative position of a DMA donor on the van der Waals surface of the $\mathrm{C} 152$ acceptor will be very important.

iii. On the time scale of the photoinduced reaction, partial diffusive reorientation could occur, but little translational motion will serve to rearrange the solvent configuration. Qualitative evidence for this is obtained by inspection of our 45 ps molecular dynamics trajectory.

iv. The near-neighbor DMA molecules have nearly identical equilibrium electrochemical properties. Because of the wide variations in orientation of DMA about the $\mathrm{C} 152$ acceptor, we can assume that most of the variation in electron-transfer rate arises from a change in the overall electronic coupling.

v. If the electronic coupling between DMA donor and C152 acceptor depends strongly on the position of the DMA relative to the coumarin molecular surface, then as the solvent orientations fluctuate, large changes will occur in the magnitude of the electronic coupling, $H_{\mathrm{DA}}$. I.e., these data are consistent with a picture where the electronic coupling is not a constant parameter, but a dynamical variable coupled to the solvation dynamics.

Comparing the rate changes for coumarin 151/DMA with toluene diluent and coumarin 152/DMA with chlorobenzene diluent, we find that the dynamical effect is pronounced in both cases. Considering further the coumarin 152 data with a $13 \%$ triphenylamine solution in benzene, we can extrapolate and assume that the electronic coupling for TPA may be stronger than initially indicated.

\section{Theoretical Results and Discussion}

The goals of our initial theoretical studies were to (A) use MD to estimate the approximate number of DMA molecules that are nearest neighbors to the $\mathrm{C} 152$, (B) examine the range

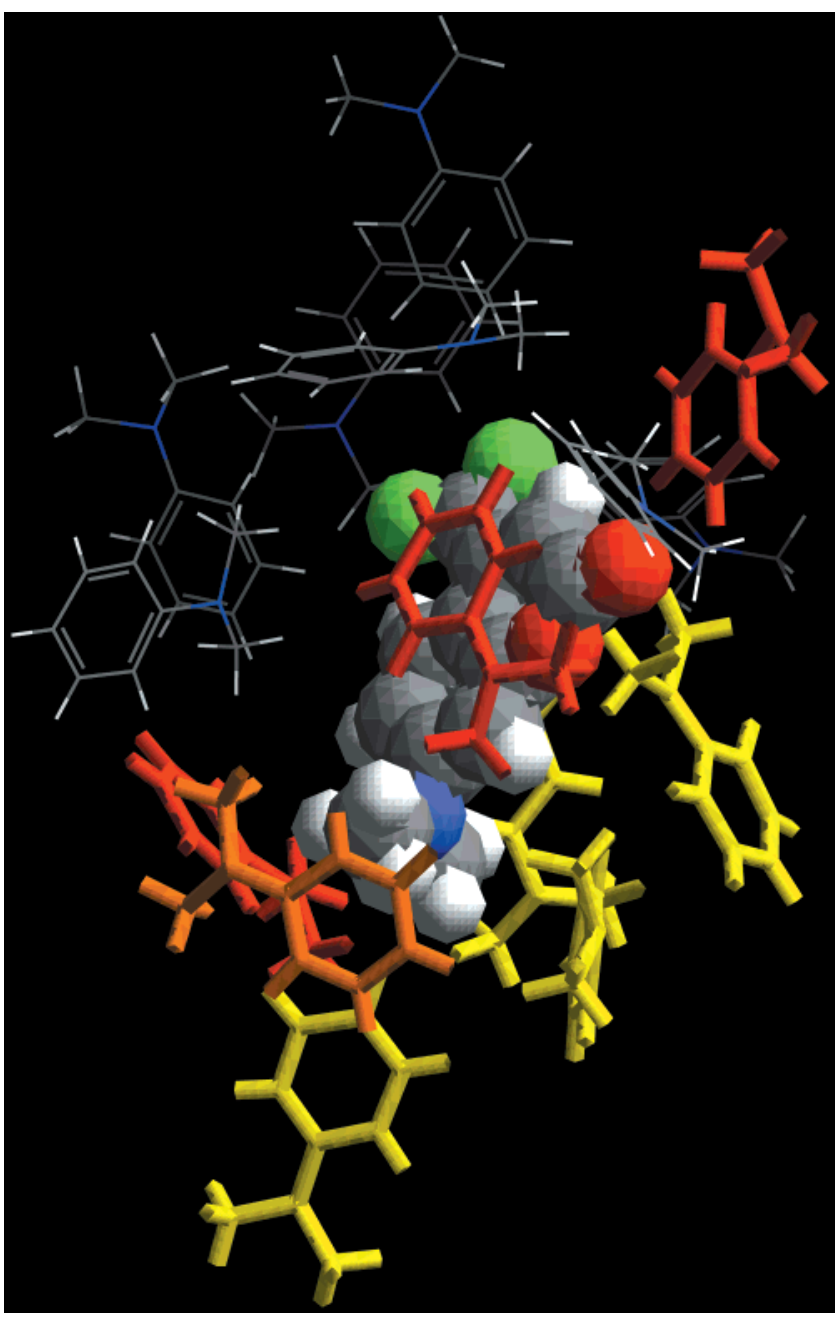

Figure 6. Coumarin 152 (C152) and nearest-neighbor DMA solvent molecules from the $40 \mathrm{ps}$ frame of trajectory 1. C152 is represented in colored spheres, gray indicating carbon, red oxygen, blue nitrogen, green fluorine, and white hydrogen. The DMA (sticks and cylinders) are coded according to the size of $H_{\mathrm{DA}}$ for electron transfer with the C152. DMAs represented as largely gray sticks indicate an $H_{\mathrm{DA}}$ value below $50 \mathrm{~cm}^{-1}$, yellow cylinders indicate a value between 50 and $100 \mathrm{~cm}^{-1}$, orange cylinders a value between 100 and $150 \mathrm{~cm}^{-1}$, and red cylinders a value greater than $150 \mathrm{~cm}^{-1}$.

of $H_{\mathrm{DA}}$ values one might expect for those in close contact, (C) attempt to relate the size of $H_{\mathrm{DA}}$ to orientation, distance, and the orbitals involved in the electron transfer, and (D) examine the variation of $H_{\mathrm{DA}}$ with time at a given position around the C152. The majority of the results presented below are from analysis of seven frames from the first trajectory of the MD simulations: one for the minimized structure and six others at 5, 10, 20, 30, 40, and 45 ps.

For the frames listed above, we found a range of 13-16 DMA within $3.5 \AA$ of at least one atom on C152. This means that, in principle, one expects upward of 15 potential electron donors in near-contact with the $\mathrm{C} 152$ acceptor, immediately following photoexcitation. Excluding local environmental effects one expects the nuclear contributions to the rate of electron transfer to be similar in all cases, and thus the dominant variation in rate with site, if there is any, will arise from the dependence of the electronic coupling element on distance and orientation. To assess this, we investigated $H_{\mathrm{DA}}$ as a function of DMA site index for the various MD time frames.

A sample frame (40 ps) is shown in Figure 6. The results from this frame are listed in Table 6 . Along with $H_{\text {DA }}$ we give 
TABLE 6: $H_{\mathrm{DA}}$ Data for C152/DMA Pairs (Nearest Neighbors) for the 40 ps Frame $^{a}$

\begin{tabular}{cccc}
\hline DMA & $\Delta E_{\text {adiabatic }}\left(\mathrm{cm}^{-1}\right)$ & $R_{\text {DA }}(\AA)$ & $H_{\text {DA }}\left(\mathrm{cm}^{-1}\right)$ \\
\hline 1 & 9280 & 11.1 & 0.4 \\
2 & 6650 & 9.5 & 1.0 \\
3 & 5610 & 7.4 & 25 \\
4 & 8080 & 8.0 & 3.0 \\
5 & 8910 & 7.4 & 28 \\
6 & 9740 & 6.9 & 127 \\
7 & 10800 & 6.2 & 566 \\
8 & 12800 & 9.5 & 70 \\
9 & 1910 & 4.0 & 779 \\
10 & 6200 & 4.6 & 60 \\
11 & 9290 & 5.7 & 85 \\
12 & 4100 & 6.5 & 85 \\
13 & 3800 & 8.4 & 266 \\
14 & 6490 & 8.6 & 6.3 \\
15 & 5540 & 9.0 & 11
\end{tabular}

${ }^{a}$ The values correspond to the various DMA in Figure 6. For details on the calculation, see the Theoretical Methods section.

the energy difference between the two adiabatic states involved in calculating $H_{\mathrm{DA}}, \Delta E_{\text {adiabatic }}$, and the GMH charge-transfer distance $R_{\mathrm{DA}}$. We find that the values of $H_{\mathrm{DA}}$ for the $15 \mathrm{DMA} /$ C152 pairs range from less than 1 to nearly $800 \mathrm{~cm}^{-1}$ for the 40 ps MD time frame.

Zusman has examined the crossover from nonadiabatic to adiabatic electron-transfer dynamics as a function of the characteristic times for solvent motion. ${ }^{57}$ Starting with the wellknown equation from the Marcus theory for nonadiabatic electron-transfer rates, ${ }^{56}$

$$
k_{\mathrm{NA}}=\frac{2 \pi}{\hbar} \frac{\left(H_{\mathrm{DA}}\right)^{2}}{\sqrt{4 \pi \lambda_{\mathrm{S}} k_{\mathrm{B}} T}} \exp \left(\frac{-\Delta G^{*}}{k_{\mathrm{B}} T}\right)
$$

Zusman $^{57}$ and others ${ }^{98,99}$ defined an adiabaticity parameter $\kappa$ describing the transition from nonadiabatic to adiabatic electron transfer. Their equation is

$$
k_{\mathrm{et}}=\frac{k_{\mathrm{NA}}}{1+\kappa} \quad \kappa=\frac{4 \pi\left(H_{\mathrm{DA}}\right)^{2} \tau_{\mathrm{S}}}{\hbar \lambda_{\mathrm{S}}}
$$

Using conventional solvent relaxation times, one predicts that the present systems become adiabatic for an electronic coupling element in the range of $40 \mathrm{~cm}^{-1}$, but with rates well below those observed here experimentally. However, this relaxation time is not appropriate to the present systems, since the fastest decay times observed here are on the order of 200 fs. Figure 7 shows results from a Zusman type analysis for several characteristic solvation times (with assumed free energy differences and solvent reorganization energies) that yield rates in the regime observed in our experiments. We see that the "adiabatic" $H_{\mathrm{DA}}$ varies considerably. As a compromise we use $150 \mathrm{~cm}^{-1}$ as our cutoff in analyzing the theoretical results. It is useful to keep in mind that larger values will lead to fewer DMA counted as "adiabatic." On the other hand, choosing this value for the MD/ GMH results presented, we find a subset of 3-5 DMA's that exhibit relatively adiabatic coupling elements, with the remainder having relatively small $H_{\mathrm{DA}}$ values.

The color coding in Figure 6 is intended to illustrate the size of $H_{\mathrm{DA}}$ for each of the DMA, where red indicates a value of $H_{\mathrm{DA}}$ greater than $150 \mathrm{~cm}^{-1}$, orange indicates $H_{\mathrm{DA}}$ in the range $100-150 \mathrm{~cm}^{-1}$, yellow the range between 50 and $100 \mathrm{~cm}^{-1}$, and gray any $H_{\mathrm{DA}}$ value less than $50 \mathrm{~cm}^{-1}$. Large $H_{\mathrm{DA}}$ values occur for a variety of DMA locations around the C152, but more than half of the DMA sites always fall in the nonadiabatic range.

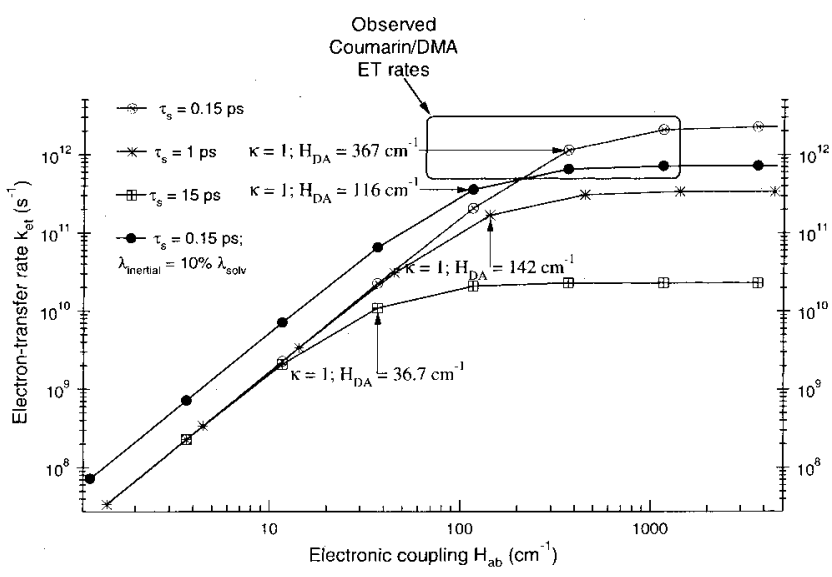

Figure 7. Zusman plots of predicted electron-transfer rate versus electronic coupling matrix element $H_{\mathrm{DA}}$. Assumptions: $T=293 \mathrm{~K}$, solvent reorganization energy $\lambda_{\text {solv }}=1210 \mathrm{~cm}^{-1}, 45$ and $\Delta G^{*}=0$. Solvent reorganization times of $0.1,1.0$, and $15 \mathrm{ps}$ are assumed, along with a fourth case for which $\lambda_{\text {solv }}=121 \mathrm{~cm}^{-1}$. The box describes the range of experimental rate values.

Since only a few of the DMAs have potentially adiabatic $H_{\mathrm{DA}}$ values for a given time, it is of interest to consider whether we can correlate our calculated $H_{\mathrm{DA}}$ values with other physical parameters. For example, the GMH $H_{\mathrm{DA}}$ values are calculated not at the transition state for electron transfer but at the fixed geometry taken from the simulation in the frame of interest. Thus, $H_{\mathrm{DA}}$ is evaluated for the geometry that is most appropriate for photoinduced electron transfer. The Condon approximation suggests that $H_{\mathrm{DA}}$ is weakly dependent on nuclear geometry ${ }^{56,100}$ and that the $H_{\mathrm{DA}}$ obtained in our calculations should be similar to that for the transition state. ${ }^{101,102}$ However, one might be concerned that there would be some trend in $H_{\mathrm{DA}}$ with adiabatic energy difference that would tend to bias our results. The results in Table 6 show that there is no discernible trend relating $H_{\mathrm{DA}}$ to $\Delta E_{\text {adiabatic }}$, with large values of $H_{\mathrm{DA}}$ occurring for both small and large adiabatic energy differences. A similar lack of correlation occurs for small values of $H_{\mathrm{DA}}$.

Another natural candidate for a parameter that might control the size of $H_{\mathrm{DA}}$ would be the charge-transfer distance, $R_{\mathrm{DA}}$. Simple models for the electronic coupling element lead us to expect a decaying exponential dependence of electronic coupling with increasing donor/acceptor separation. ${ }^{56,100}$ Indeed, there is no question that as the distance between the donor and acceptor increases in the solution, there will be a tendency for the coupling to decrease. However, within the sample of C152 nearest neighbors, we find that the correlation between distance and $H_{\mathrm{DA}}$ only obtains in a qualitative sense, with some of the longer distances producing sizable $H_{\mathrm{DA}}$ values. Analysis of the GMH results provides a qualitative estimate of the variability in $R_{\mathrm{DA}}$ for each of the DMA solvent neighbors. For a given DMA molecule, values of $R_{\mathrm{DA}}$ obtained from $\mathrm{GMH}$ analysis (of the $0,5,10,20,30,40$, and 45 ps time slices from the MD trajectory) fluctuate on average by $3.9 \AA$.

Perhaps a stronger indicator of the size of $H_{\mathrm{DA}}$ is the position of the DMA relative to the $\mathrm{C} 152$, and in particular, the position of the DMA in relation to the HOMO of the C152 (the orbital which accepts the electron in our model). Figure 8 presents the HOMOs of C152 and DMA obtained from a ZINDO RHF calculation. It is seen that the HOMO of DMA is $\pi$-like and is distributed fairly uniformly over the whole molecule. Note that the HOMO is not largely concentrated on the amine group of the C152. The C152 HOMO is also largely $\pi$-like, extending over most of the ring $\pi$ system and having significant density near the dimethylamino and the carboxyl groups. DMAs with 

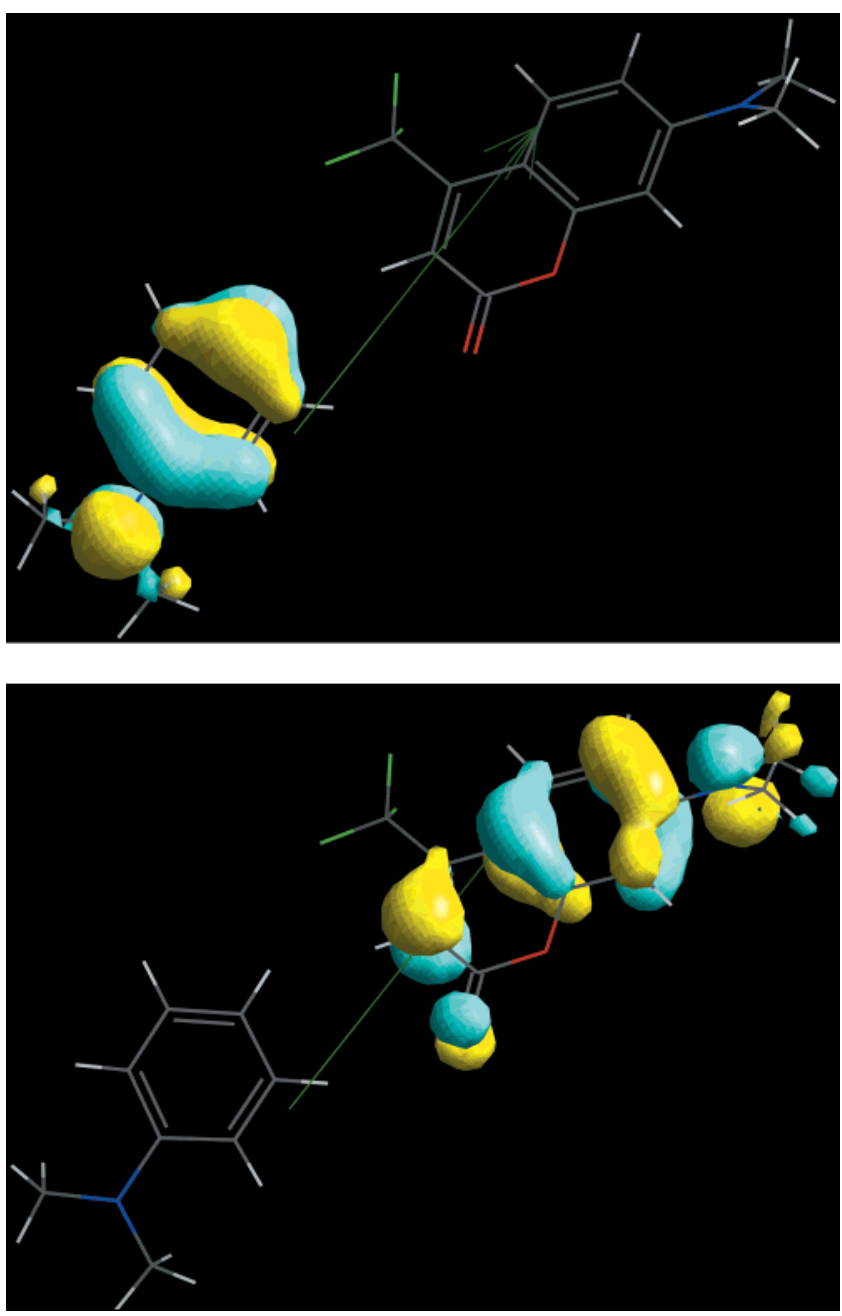

Figure 8. (a, Top) HOMO of DMA. This represents the donor orbital in the electron-transfer process. (b, Bottom) HOMO of C152. This represents the acceptor orbital in electron transfer following photoexcitation of the $\mathrm{C} 152$.

large $H_{\mathrm{DA}}$ values are indeed found at a variety of locations around the $\mathrm{C} 152$, consistent with the delocalized nature of the donor and acceptor orbitals. On the other hand, the C152 HOMO has little to no density on the trifluoromethyl group, and we find that DMAs in this region never yield large $H_{\mathrm{DA}}$ values. Reasoning further on the basis of the orbital shapes and orientations that might yield maximal overlap, one expects that face-to-face orientations will tend to yield larger $H_{\mathrm{DA}}$ values than T-shaped orientations, and this is indeed the case.

We present in Figure $9 \mathrm{a}-\mathrm{f}$ the results of calculations of $H_{\mathrm{DA}}$ for all of the other nearest neighbors for the frames from the dynamics sampled at the minimized structure, 5, 10, 20, 30, and $45 \mathrm{ps}$, with color coding identical to that of Figure 6. The results of these figures reveal several interesting features. First, it is seen that there are usually more adiabatic donors in the trajectory frames than in the minimized frame. While one might suspect that this is due to the lack of pressure equilibration in the early stages of the trajectory, it is found that the number of adiabatic (red) DMA does not decrease over time, thus pressure changes do not appear to be a significant factor in this range. Rather, it is likely that since thermal motion will yield some molecular pairs that tend to ride up the repulsive wall of the intermolecular potential, there is a strong possibility of greater overall interactions and potentially larger values of $H_{\mathrm{DA}}$. Second, as in the 40 ps frame (Figure 6), we find that relatively strong interactions (large $H_{\mathrm{DA}}$ ) for C152/DMA occur at almost all locations around the $\mathrm{C} 152$, with the exception of DMA molecules in the region of the trifluoromethyl group and those perpendicular to the $\mathrm{C} 152$ ring plane.

These figures also reveal interesting information about the time dependence of $H_{\mathrm{DA}}$. For example, focusing on the $10 \mathrm{ps}$ frame, one sees an orange DMA on the lower left portion of Figure 9c. At 20 ps this DMA has moved slightly and the value of $H_{\mathrm{DA}}$ has fallen into the "yellow" range. At 30 ps the coupling element falls below $50 \mathrm{~cm}^{-1}$ but grows again at $40 \mathrm{ps}$. Thus, while one might want to think about "sites" around the C152 as being strongly or weakly interacting, it is clear that subtle changes in geometry for nearest-neighbor DMA/C152 pairs can lead to substantial changes in the size of $H_{\mathrm{DA}}$ and thus the estimated rate of reaction. Alternatively, one can focus on the red DMA that is cofacial with the C152. Even though it moves somewhat over the time period, its coupling element remains large. Obviously, one can use the figures to follow other DMA as well. For example, significant orientational motion and $H_{\mathrm{DA}}$ changes occur for DMAs located near the dimethylamino group on the $\mathrm{C} 152$. Of course, all of the predicted behavior is based on the use of ZINDO wave functions, using a rather restricted $\mathrm{CI}$, and when it becomes possible to use more exact wave functions, the details of the results presented here may change. However, on the basis of our test calculations we expect the general trends observed here to persist. For example, using an expanded CI space for the 40 ps frame, we obtain three red, two orange, and three yellow DMA, as opposed to three, one, and four, respectively, in the set we use, with excellent correspondence between the colored species in each frame. Thus, we still expect a wide range of $H_{\mathrm{DA}}$ values to be observed for the set of nearest neighbors.

Small changes in geometry can significantly alter $H_{\mathrm{DA}}$ for C152/DMA pairs where $H_{\mathrm{DA}}$ is near the adiabatic threshold. Further evidence for this is obtained in the results of Figure 10 , where a single DMA is followed over nearly $1 \mathrm{ps}$, calculating $H_{\mathrm{DA}}$ every $25 \mathrm{fs}$. We selected the DMA nearest the C152 dimethylamino group, because we expected both large $H_{\text {DA }}$ values and sensitivity of $H_{\mathrm{DA}}$ to inertial fluctuations for this solvent location. In Figure 10 we have drawn a line indicating a coupling value of $150 \mathrm{~cm}^{-1}$ to guide the eye. The relative orientation between DMA and C152 rings planes is about $45^{\circ}$, as indicated by the inset molecular models. It is seen that even on a time scale of $25 \mathrm{fs}$ steps there are significant fluctuations in the electronic coupling and that on occasion the coupling element becomes quite large. Analysis of the results shows that the local motions of the $\mathrm{C} 152$ chromophore can induce state mixings that have dramatic effects on the calculated electronic coupling, leading to both small values (during the period 280$300 \mathrm{fs}$ ) and large values (during 800-900 fs). Further theoretical work needs to be performed to examine the details of the state descriptions and the effects on the estimated coupling, but the present results suggest $H_{\mathrm{DA}}$ can be strongly affected by small intramolecular and solvent motions and thus is truly a dynamical variable for this system. We note that Neyhart et al. speculated that solvent librations could induce large changes in $H_{\mathrm{DA}}$ for electronic coupling across the bridging ligand for intramolecular electron-transfer in binuclear $\mathrm{Ru} / \mathrm{Os}$ systems. ${ }^{103}$

Intramolecular motions that one might expect to correlate with $H_{\mathrm{DA}}$ are the torsions and inversions of the dimethylamino group on either DMA or C152. In results from our third trajectory we have found a DMA molecule that is in close proximity to $\mathrm{C} 152$ and is undergoing inversion. We have examined $H_{\mathrm{DA}}$ as a function of this motion. In Figure 11 the range of motions is shown both for the DMA and the C152. On the DMA the 

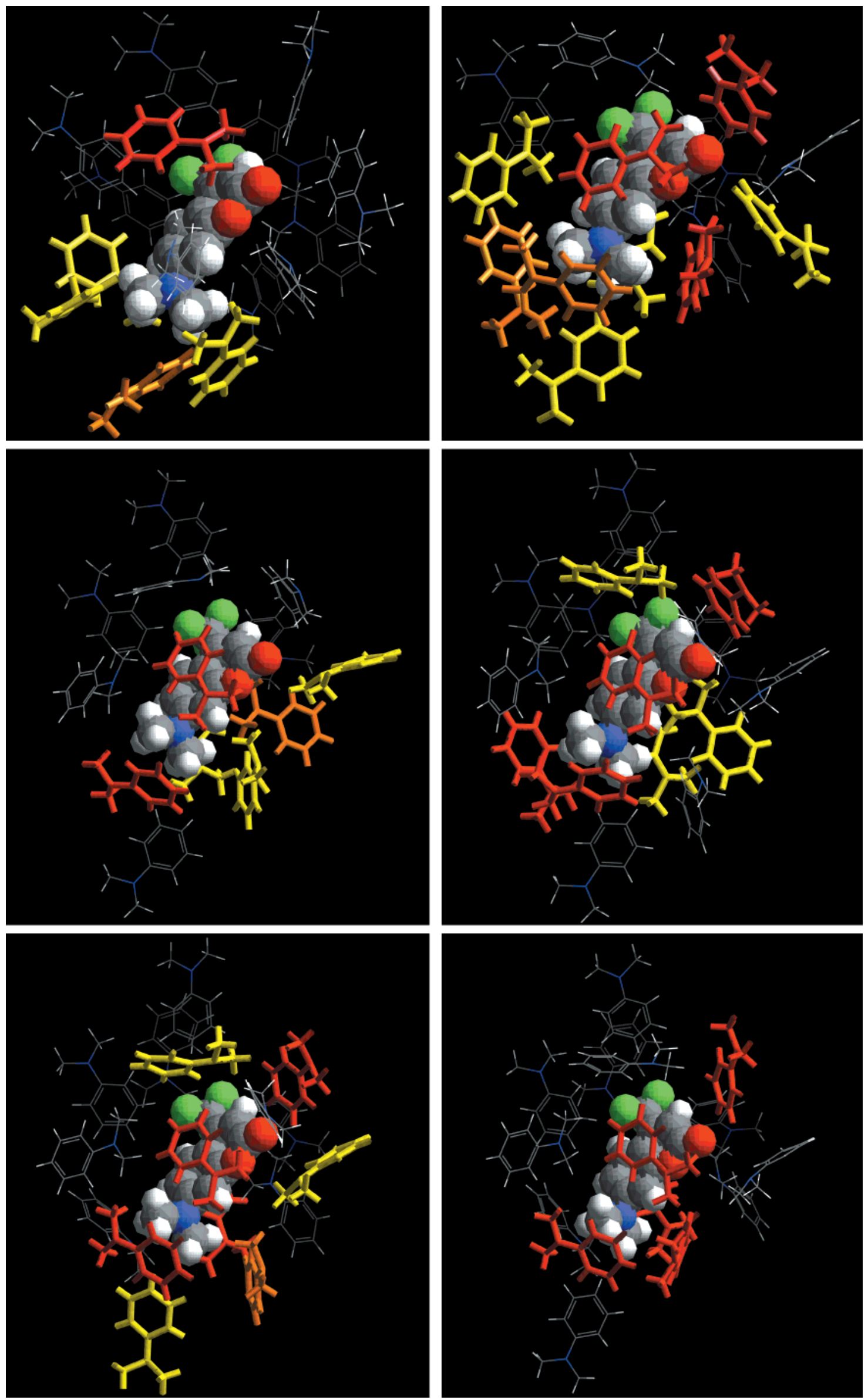

Figure 9. C152 and nearest neighbors from various time frames from trajectory 1 (color-coded as in Figure 6): (a) minimized structure; (b) 5 ps, (c) $10 \mathrm{ps}$, (d) $20 \mathrm{ps}$, (e) $30 \mathrm{ps}$, and (f) $45 \mathrm{ps}$. Sections (a)-(f) are arrayed from left to right, top to bottom. 


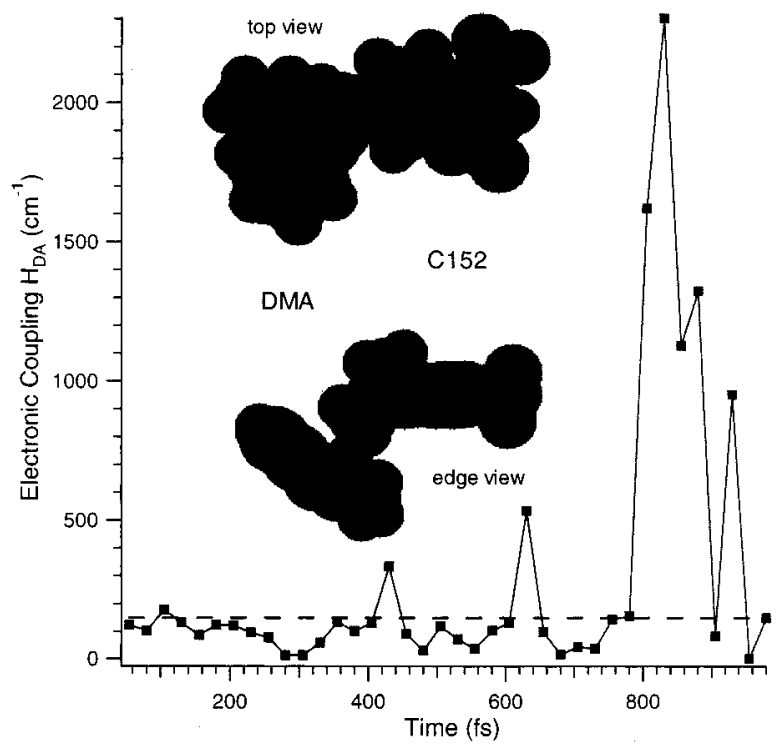

Figure 10. $H_{\mathrm{DA}}$ as a function of time for a single DMA-C152 pair from trajectory 2, sampled every 25 fs. Models for the van der Waals surfaces of the specific C152/DMA pair are shown to give an idea of the relative orientations. The DMA is located near the $\mathrm{C} 152$ dimethylamino group, with about a $45^{\circ}$ angle between the ring planes. See text for details of the calculation.

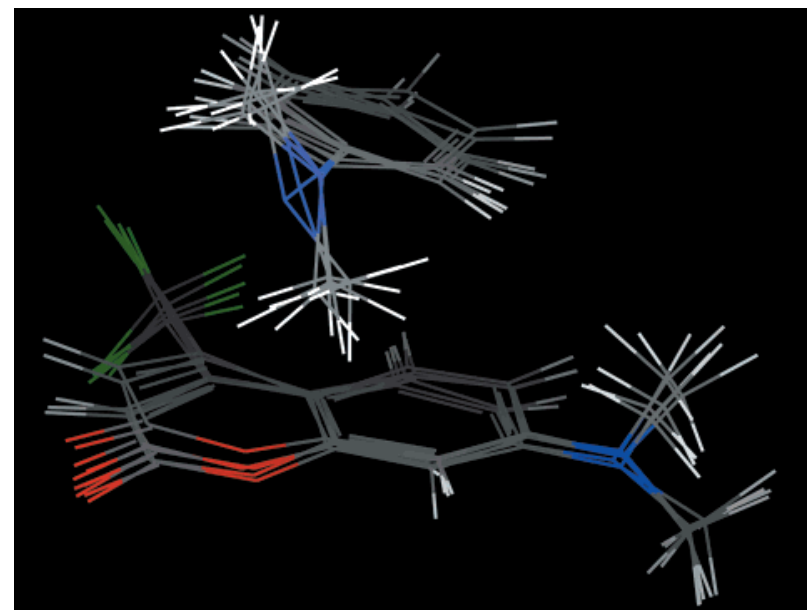

Figure 11. Representative geometries for the C152/DMA pair examined in Table 7, where the DMA group is undergoing inversion. Frames taken from trajectory 3 .

TABLE 7: $H_{\mathrm{DA}}$ Data C152/DMA Electron Transfer for Various Inversion Angles of DMA As shown in Figure $10^{a}$

\begin{tabular}{ccccc}
\hline${\text { time }(\mathrm{fs})^{b}}^{b}$ & ${\text { angle }(\mathrm{deg})^{c}}^{c}$ & $\Delta E_{\text {adiabatic }}\left(\mathrm{cm}^{-1}\right)$ & $R_{\mathrm{DA}}(\AA)$ & $H_{\mathrm{DA}}\left(\mathrm{cm}^{-1}\right)$ \\
\hline 400 & 35 & 3240 & 3.8 & 321 \\
440 & 20 & 1670 & 3.9 & 545 \\
500 & 11 & 1376 & 3.6 & 270 \\
550 & 29 & 333 & 3.3 & 124 \\
590 & -51 & 3178 & 3.2 & 592
\end{tabular}

${ }^{a}$ See text for details on the calculation. ${ }^{b}$ Time of this frame in trajectory $3 .{ }^{c}$ Inversion angle at the DMA nitrogen.

dominant motion is the inversion of the amine group, but it is also seen that the $\mathrm{C} 152$ ring undergoes significant flexing during this period of time. Table 7 shows the $H_{\mathrm{DA}}$ values for this sequence. From these data, it is tempting to interpret the inversion motion as being responsible for significant modulation of $H_{\mathrm{DA}}$, but inspection of the intermediate steps (not shown) shows similar variation in $H_{\mathrm{DA}}$, whereas there is essentially no inversion motion accompanying these changes. Rather, the predominant motion is the flexing of the $\mathrm{C} 152$ ring.
Normal coordinate calculations were done for both $\mathrm{C} 152$ and DMA using the PM3 force field. ${ }^{104-106}$ For the C152 molecule, the lowest frequency modes from this model occur at these frequencies: $34,45,70,88,124,126,151,165,208$, and 229 $\mathrm{cm}^{-1}$. There is no pure inversion mode at the $\mathrm{C} 152$ nitrogen, though the eighth mode at $165 \mathrm{~cm}^{-1}$ comes closest to matching this description. The two lowest frequency modes at 34 and 45 $\mathrm{cm}^{-1}$ show a large degree of torsional character about the nitrogen atom, as do the 126 and $151 \mathrm{~cm}^{-1}$ modes. Large coumarin ring distortions occur for the modes at 70 and $88 \mathrm{~cm}^{-1}$. It is especially interesting to note that these $\mathrm{C} 152$ ring vibrations have good spectral overlap with the intermolecular librations for liquid DMA. ${ }^{49}$

The smaller DMA phenyl ring is far more rigid. The first four normal modes of DMA from the PM3 calculation will be populated at room temperature and occur at $41,133,153$, and $200 \mathrm{~cm}^{-1}$. Assignments for these four modes are: $41 \mathrm{~cm}^{-1}$, antisymmetric dimethylamino/phenyl twist; $133 \mathrm{~cm}^{-1}$, symmetric dimethylamino/phenyl bend; $153 \mathrm{~cm}^{-1}$, antisymmetric methyl twist; and $200 \mathrm{~cm}^{-1}$, symmetric dimethylamino/phenyl bend, with methyl twist.

We see that the mutual orientation of the electron donor and acceptor can have a significant effect on the value of $H_{\mathrm{DA}}$. Consider Figure 6 again, where one observes the C152 nearly edge-on and can thus see the DMA neighbors nearest both faces of the C152. On one face of the C152 there is a red DMA in a cofacial orientation with the $\mathrm{C} 152$, while on the other side three yellow DMAs assume approximately T-shaped orientations with the $\mathrm{C} 152$. The former orientation would be expected to produce larger overlap of the donor and acceptor orbitals, and we generally find the face-to-face orientation to yield large values of $H_{\mathrm{DA}}$. The actual values of $H_{\mathrm{DA}}$ (in $\mathrm{cm}^{-1}$ ) for the four DMA molecules that are on either face of the $\mathrm{C} 152$ are 780 (red), 85 and 85 (orange), and 60 (yellow). The GMH charge-transfer distances (in $\AA$ ) for these cases are 4.0 (red), 5.7 and 6.5 (orange), and 4.6 (yellow). In addition, the other red DMAs in this frame have charge-transfer distances ranging from 6.2 to $8.3 \AA$. Thus, the distance between charge centroids for the initial and final diabatic states has little correlation with the relative size of $H_{\mathrm{DA}}$ for these nearest-neighbor pairs. It is clear that orientation plays a large role in determining $H_{\mathrm{DA}}$ for the $\mathrm{C} 152 /$ DMA pairs.

The total picture from our GMH results (from Trajectory 1) for the electronic coupling between first solvent layer DMA molecules and a C152 acceptor is summarized in Figure 12. The electronic coupling matrix elements $H_{\mathrm{DA}}$ for each DMA solvent (which is in van der Waals contact with the central C152 solute) are plotted, using the data from all seven time-slice frames from the MD trajectory. For the collection of the 15 near-neighbor DMA molecules surrounding the C152 solute, we see in Figure 12 that the electronic coupling $H_{\mathrm{DA}}$ spans the range from 0.04 to $2013 \mathrm{~cm}^{-1}$. Because of the anisotropies in both molecular shapes and orientational distributions, the donor-acceptor separations $R_{\mathrm{DA}}$ range from 2.6 to $11.4 \AA$.

It is important to use a sensible definition for $R_{\mathrm{DA}}$, because each of the 15 DMA molecules has one or more atom pairs between $\mathrm{C} 152$ and DMA with a center-to-center distance of $\leq 3.5 \AA$. In Figure 12, we find a variation in $H_{\mathrm{DA}}$ of about a factor of 100 for a given donor-acceptor distance $R_{\mathrm{DA}}$. Though the scatter in this plot is large, we can nevertheless fit the rough exponential dependence of $H_{\mathrm{DA}}$ on separation $R_{\mathrm{DA}}$ to obtain a slope of $0.885 \AA^{-1}$. Since the Marcus theory for electron-transfer reaction rates predicts a rate that will vary as the square of the electronic coupling matrix element, ${ }^{56}$ twice the slope from our 


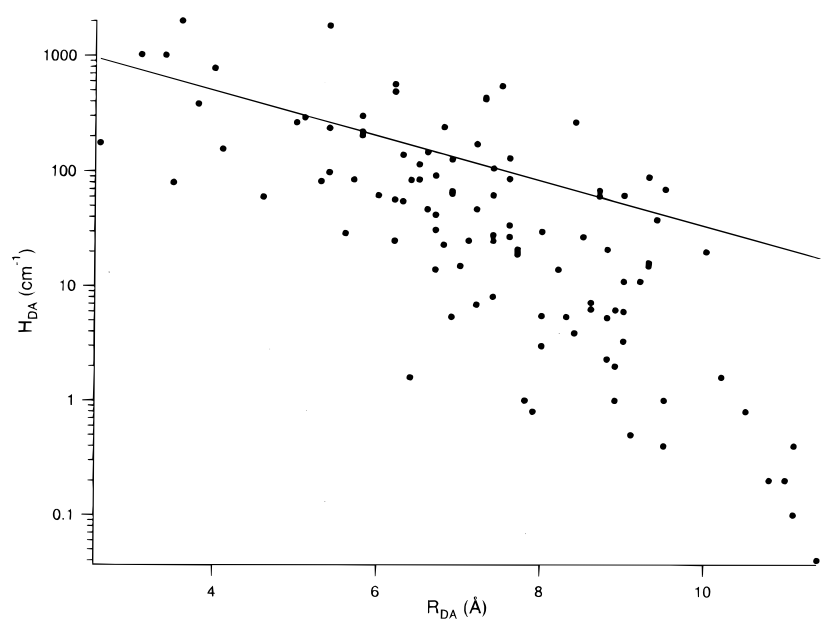

Figure 12. Plot of the sum total of GMH computational results for seven time slices from the MD simulations: Energy minimized ( $0 \mathrm{ps})$, $5,10,20,30,40$, and $45 \mathrm{ps}$. The electronic coupling matrix element $H_{\text {DA }}$ is plotted on a semilog ordinate scale in energy units of $\mathrm{cm}^{-1}$. The least-squares fit of $\ln \left(H_{\mathrm{DA}}\right)$ versus $R_{\mathrm{DA}}$ gives a slope of -0.885 $\AA^{-1}$, intercept of $21368 \mathrm{~cm}^{-1}$, and a poor correlation coefficient of $R^{2}=0.55$.

plot of $\ln \left(H_{\mathrm{DA}}\right)$ versus donor-acceptor separation $R_{\mathrm{DA}}$ (Figure 12) provides the value $\beta=1.77 \AA^{-1}$. Though values for $\beta$ vary widely in many experimental and theoretical studies, this value is substantially larger than found for some experimental systems, but in reasonable agreement with the value of $\beta=2.0 \AA^{-1}$ reported for electron-transfer through water in another GMH study. ${ }^{102}$ The centroid of distance values for Figure 12 is in the range of $6.5-7.0 \AA$. We can extrapolate from the exponential fit to our data to see that for twice this distance, i.e., for DMA molecules in the second solvent layer, $H_{\mathrm{DA}}$ values will be so small as to never become adiabatic, even with a generous fluctuation factor of up to 100. Should super exchange mechanisms for electron transfer from DMA donors in the second solvent layer occur, a smaller value of $\beta$ (e.g., $1.0 \AA^{-1}$ ) may result. Even in this case, we still expect transfer rates from the second layers to be negligibly small. Thus, our initial assumption that all of the chemistry would occur from DMA donor molecules within the first solvent layer appears to have been justified.

\section{Summary and Conclusions}

The present work extends the discussion of ultrafast solventas-donor electron-transfer systems, using various coumarins as electron acceptors and substituted anilines as donors. The variety of solvents and coumarins used allows one to observe the sensitivity of the fastest components of the rate to energetic and structural parameters of both donor and acceptor. The most novel characteristic of the present experimental results is the ubiquitous $100-150$ fs rise time that precedes the electrontransfer event. This rise time is similar in duration to librational periods observed in Kerr experiments on DMA, and suggests that even the very fast electron-transfer rates observed here require modest solvent reorganization before electron transfer occurs. However, there are also a number of other low-frequency motions occurring in our substituted coumarin/aniline systems. In particular, (dimethyl)amino torsions or inversions can occur on both donor and acceptor. Also, large amplitude ring flexing vibrations of the coumarin rings are observed in our normalmode quantum calculations.

Since we prepare the acceptor excited state to be within 500 $\mathrm{cm}^{-1}$ of the $0-0$ absorption transition, there is little intramo- lecular excess vibrational energy to drive the reaction. In terms of the Sumi-Marcus model, ${ }^{68}$ if there were greater intramolecular vibrational excitation, the reaction could occur more nearly "perpendicular" to the solvent coordinate than it does here. This suggests that it would be interesting to study the complete emission profile as a function of excitation wavelength, resolving both wavelength and temporal dimensions. It could be possible to examine how electron-transfer rates depend on both the intramolecular vibrational relaxation and on the solvent reorganization response.

Zinth and co-workers have shown a number of interesting characteristics for the photoinduced electron-transfer reaction of oxazine 1 with DMA. ${ }^{95,96,107,108}$ They observed that the electron-transfer dynamics extracted from transient absorption spectra (with sub-20 fs time resolution) were biexponential, with time constants of 30 and 80 fs. It is not at all surprising that they observed coherent vibrational dynamics in the transients for the oxazine 1 excited state, as these impulsive Raman wave packet dynamics are ubiquitous in femtosecond absorption transients. However, it is indeed surprising that they also observed oscillatory wave packet dynamics in transients detecting the oxazine 1 radical-cation product state. This indicates that the oxazine 1/DMA system must also be in the adiabatic or strong coupling electron-transfer regime, as we have found to be the case for C152/DMA and, by analogy, for all of the coumarin/DMA systems we have studied.

Our experimental work on $\mathrm{C} 152$ and various electrondonating solvents, and on DMA quenching of the six coumarins, shows the subtle effects of substituents on the rate of transfer. This area has been explored in great detail by Yoshihara and co-workers..$^{37-53} \mathrm{We}$ find excellent agreement between our C152/DMA results and theirs, and good agreement with their earlier results on C151/DMA. ${ }^{40}$ More recent work by Shirota et al. indicates that the C151/DMA photoreaction is also slightly biexponential in rate ${ }^{50}$ However, our results for the particular case of C343 call into question the assumption of a TICT-like mechanism for the electron-transfer event. Finally, the results of our DMA dilution experiments give conflicting results concerning whether solvent molecules are equivalent in their ability to react rapidly with the coumarin. If this were the case, one would expect a linear decrease in the amplitude of the fast component of the reaction with donor concentration, and to a good extent, this is what is observed for the fast component of the electron-transfer dynamics in the C151/DMA case. On the other hand, in the C152/DMA case we see a slower-thanexpected decrease of the amplitude of the fastest component with concentration, but this is accompanied by a considerable decrease in the rate of the fast component. It is possible this decrease in rate may indicate an even faster decrease in the fraction of molecules reacting with subpicosecond rates, but the fits to the experimental data may not be sufficient to extract this component at high dilutions. If this is true, it is not clear what sort of cooperative effects the dilution has on the overall concentration of "fast" reacting species. Of course, yet another possibility is that C151/DMA pairs may be more likely to be adiabatic than C152/DMA pairs.

The picture described in this article is in overall agreement with the most recent work of Xu et al. ${ }^{109}$ They used 3-pulse photon echo peak-shift (3PEPS) and transient grating experiments to measure electron-transfer rates and solvent dynamics for the case of rhodamine $6 \mathrm{G}$ as the excited-state acceptor, with DMA and $N, N$-diethylaniline (DEA) as the solvent/donors. Forward charge separation and backward geminate charge recombination time constants are 85 fs and 4.0 ps for DMA 
and $160 \mathrm{fs}$ and $6.9 \mathrm{ps}$ for DEA, respectively. ${ }^{109}$ Prior to the very rapid electron-transfer event, they observe characteristics of inner- and outer-sphere reorganization from both the rhodamine $6 \mathrm{G}$ vibrational modes and solvent dynamics. Additionally, their experimental methods allow for estimation of the ground-state cooling and relaxation after back electron transfer; these time constants are 19 ps (DMA) and 50 ps (DEA). It seems to be clear that these results are consistent with the picture we have developed above.

Using computer simulations to probe the C152/DMA system, we have begun to answer a variety of basic questions. On the basis of dynamics calculated with the force fields used in the present study, it appears that $\mathrm{C} 152$ has from 13 to 16 nearestneighbor DMA molecules in contact with it. While it is possible that electron transfer could occur to the next layer of solvent molecules, the expected decrease in electronic coupling with distance $\left(\beta=1.8 \AA^{-1}\right)$ will produce significantly lower rates than the slowest observed here. Though there is enormous scatter in the semilog plot of $H_{\mathrm{DA}}$ versus $R_{\mathrm{DA}}$, it is reassuring that there is an overall exponential decrease of $H_{\mathrm{DA}}$ with distance (yielding a plausible value of $\beta$ ) and that this $\beta$ value precludes electron donation from any DMA solvent molecules outside of the first solvent layer.

Focusing on the nearest-neighbor DMA molecules in the computer simulations reveals considerable heterogeneity in their electronic coupling to the $\mathrm{C} 152$ acceptor and thus in their conditional probabilities for becoming successful electron donors. Examining the various MD time slices shows that there will be 2-5 DMAs with adiabatic values of $H_{\mathrm{DA}}$ at a given instant. These DMA molecules have the highest probability for contributing to the fastest components of the overall electrontransfer rates. The adiabatic cutoff of $150 \mathrm{~cm}^{-1}$ used here is based on a simple Zusman analysis using an average solvent relaxation time. If we used the very fastest inertial solvation time constants (thus mandating a larger value of $H_{\mathrm{DA}}$ to be classed "adiabatic"), the number of adiabatic DMAs would have dropped to $1-3$ on average. The theoretical results indicate that while there are many potential donors surrounding a given $\mathrm{C} 152$, there are in fact only a few that can undergo rapid electron transfer.

We have analyzed the electronic coupling elements between various DMA/C152 pairs on the basis of the orbitals involved in the electron-transfer process and their overlaps. In our calculations, using simple ZINDO CI wave functions, we find that the acceptor orbital on the $\mathrm{C} 152$ is largely delocalized. There exists the possibility for DMAs (near almost any site around the $\mathrm{C} 152$ ) to yield large values of the coupling $H_{\mathrm{DA}}$, with the exception of those near the trifluoromethyl group. However, the orientation at a site is perhaps the most important factor in determining the relative size of the coupling. Since both the donor and acceptor orbitals are largely $\pi$-like, it is found that face-to-face-orientations tend to produce very large values of $H_{\mathrm{DA}}$, while $\mathrm{T}$-shaped geometries of nearest neighbors yield generally smaller values.

Significantly more theoretical work needs to be done on these systems before a complete understanding of the dynamics will be achieved. As noted above, the wave functions used for the GMH analysis are relatively simple ones. There are indications that the CI states used here, at least at the ZINDO level, may be missing contributions from a second zeroth-order configuration. Test calculations suggest that the overall coupling (and the conclusions we draw from them here) will be unaffected by use of the expanded CI, but more detailed calculations need to be performed to better understand the actual character of the
C152 excited state prepared experimentally. Such studies will also be helpful in examining the sensitivity of the excited state to the geometry of $\mathrm{C} 152$ and to determine whether the extreme variations observed in the $H_{\mathrm{DA}}$ data in Figure 12 are realistic. Last, from the perspective of sampling uncorrelated molecular orientations, the dynamics runs we used were relatively short and used relatively few solvent molecules. We would like to extend the simulations to significantly longer times and to use more DMA solvent in order to more accurately probe specific orientational effects on the donor/acceptor electronic coupling.

Of course, the present theoretical analysis makes the drastic assumption that all nearest-neighbors have equivalent activation free energies, and thus that only the value of $H_{\mathrm{DA}}$ plays a role in determining the relative rates in the system. This is unquestionably an oversimplification, as shown by Shirota et al. ${ }^{51}$ Further work, perhaps using continuum dielectric models, may be able to address local differences in solvent reorganization energies (although the fast time scales observed here may make these estimates largely irrelevant to the rates). On the other hand, simulations beginning with equilibrated distributions around the ground-state $\mathrm{C} 152$, followed by an instantaneous change to a charge distribution characteristic of the $\mathrm{C} 152$ excited state, may reveal characteristic motions of the local DMA that could correspond more precisely to the rise times observed. Finally, simulations using mixtures of DMA with nonreactive solvent will allow us to better understand the dilution data.

Acknowledgment. E.W.C. wishes to thank his former Brookhaven colleagues for their assistance with different aspects of this work: Mei Chou for help with the solvent purification; Rich Rozensky, Connie Kohler, and Dick Becker for fabrication of instrument components; and Drs. Bruce Brunschwig, Etsuko Fujita, James Wishart, and Norman Sutin for a number of helpful discussions. This research was carried out at Brookhaven National Laboratory, Rutgers University, and Harvey Mudd College. Brookhaven work was supported by contract DEAC02-98CH10884 with the U.S. Department of Energy and supported by its Division of Chemical Sciences, Office of Basic Energy Sciences (E.W.C.). R.J.C. acknowledges support of this research by grants from the National Science Foundation (CHE9731634) and the donors of the Petroleum Research Fund. D.K. was supported by a grant from the National Science Foundation for Research Experiences for Undergraduates (CHE-9732111). We thank Dr. Hideaki Shirota and Dr. Yeung-gyo Shin (Rutgers) for helpful discussions and a careful reading of the manuscript.

Supporting Information Available: Tables of $H_{\mathrm{DA}}$ data for C152/DMA pairs and absorption/emission spectral data. This information is available free of charge via the Internet at http:// pubs.acs.org.

\section{References and Notes}

(1) Electron Transfer-From Isolated Molecules to Biomolecules, Part 1; Jortner, J.; Bixon, M., Eds.; John Wiley: New York, 1999; Vol. 106.

(2) Electron Transfer-From Isolated Molecules to Biomolecules, Part 2; Jortner, J.; Bixon, M., Eds.; John Wiley: New York, 1999; Vol. 107.

(3) Barbara, P. F.; Meyer, T. J.; Ratner, M. A. J.Phys. Chem. 1996, $100,13148-13168$.

(4) Closs, G. L.; Calcaterra, L. T.; Green, N. J.; Penfield, K. W.; Miller, J. R. J. Phys. Chem. 1986, 90, 3673-83.

(5) Closs, G. L.; Miller, J. R. Science 1988, 240, 440-447.

(6) Roest, M.; Lawson, J. M.; Paddon-Row: M. N.; Verhoeven, J. W. Chem. Phys. Lett. 1994, 230, 536-542.

(7) Schuddeboom, W.; Scherer, T.; Warman, J. M.; Verhoeven, J. W. J. Phys. Chem. 1993, 97, 13092-13098.

(8) Verhoeven, J. W.; Wegewijs, B.; Kroon, J.; Rettschnick, R. P. H.; Paddon-Row, M. N.; Oliver, A. M. J. Photochem. Photobiol. A: Chem. 1994, 82, 161-170. 
(9) Hung, S.-C.; Macpherson, A. N.; Lin, S.; Liddell, P. A.; Seely, G. R.; Moore, A. L.; Moore, T. A.; Gust, D. J. Am. Chem. Soc. 1995, 117 $1657-1658$

(10) Macpherson, A. N.; Liddell, P. A.; Lin, S.; Noss, L.; Seely, G. R. DeGraziano, J. M.; Moore, A. L.; Moore, T. A.; Gust, D. J. Am. Chem. Soc. 1995, 117, 7202-7212.

(11) Wiederrecht, G. P.; Niemczyk, M. P.; Svec, W. A.; Wasielewski, M. R. J. Am. Chem. Soc. 1996, 118, 81 .

(12) Kumar, K.; Lin, Z.; Waldeck, D. H.; Zimmt, M. B. J. Am. Chem. Soc. 1996, 118, 243.

(13) Cave, R. J.; Newton, M. D.; Kumar, K.; Zimmt, M. B. J. Phys.l Chem. 1995, 99, 17501-17504.

(14) Pöllinger, F.; Heitele, H.; Michel-Beyerle, M. E.; Anders, C.; Futscher, M.; Staab, H. A. Chem. Phys. Lett. 1992, 198, 645-652.

(15) Bixon, M.; Jortner, J.; Cortes, J.; Heitele, H.; Michel-Beyerle, M. E. J. Phys. Chem. 1994, 98, 7289-7299.

(16) Wynne, K.; Lecours, S. M.; Galli, C.; Therien, M. J.; Hochstrasser, R. M. J. Am. Chem. Soc. 1995, 117, 3749-3753.

(17) Wen, X.; Zhang, R.; Spears, K. G. J. Phys. Chem. 1996, 100.

(18) Kulinowski, K.; Gould, I. R.; Myers, A. B. J. Phys. Chem. 1995, 99, 9017-9026.

(19) Wynne, K.; Reid, G. D.; Hochstrasser, R. M. J. Chem. Phys. 1996 $105,2287-2297$.

(20) Wynne, K.; Hochstrasser, R. M. Coherence and adiabaticity in ultrafast electron transfer. Electron Transfer-From Isolated Molecules to Biomolecules; 1999; Vol. 107, Part 2, pp 263-309.

(21) Isied, S. S.; Ogawa, M. Y.; Wishart, J. F. Chem. Rev. 1992, 92 , $381-394$.

(22) Ogawa, M. Y.; Moreira, I.; Wishart, J. F.; Isied, S. S. Chem. Phys. 1993, 176, 589-600

(23) Jones, I.; G.; Lu, L. N.; Fu, H.; Farahat, C. W.; Oh, C.; Greenfield,

S. R.; Gosztola, D. J.; Wasielewski, M. R. J. Phys. Chem. B 1999, 103.

(24) Wishart, J. F.; van Eldik, R.; Sun, J.; Su, C.; Isied, S. S. Inorg. Chem. 1992, 31, 3986-3989.

(25) Mutz, M. W.; McLendon, G. L.; Wishart, J. F.; Gaillard, E. R.; Corin, A. F. Proc. Natl. Acad. Sci. U.S.A. 1996, 93, 9521-9526.

(26) Wishart, J. F.; Sun, J.; Cho, M.; Su, C.; Isied, S. S. J. Phys. Chem. B 1997, 101, 687-693.

(27) Skov, L. K.; Pascher, T.; Winkler, J. R.; G. H. B. J. Am. Chem. Soc. 1998, 120, 1102-1103.

(28) Berglund, J.; Pascher, T.; Winkler, J. R.; Gray, H. B. J. Am. Chem. Soc. 1997, 119, 2464-2469.

(29) Gray, H. B.; Winkler, J. R. Аnnu. Rev. Biochem. 1996, 35, $537-$

(30) Shifman, J. M.; Moser, C. C.; Kalsbeck, W. A.; Bocian, D. F.; Dutton, P. L. Biochemistry 1998, 37, 16815-16829.

(31) Maiti, S.; Walker, G. C.; Cowen, B. R.; Pippenger, R. S.; Moser, C. C.; Dutton, P. L.; Hochstrasser, R. M. Biophys. J. 1994, 66, A272-A272.

(32) Lewis, F. D.; Wu, T.; Zhang, Y.; Letsinger, R. L.; Greenfield, S.

R.; Wasielewski, M. R. Science 1997, 277, 673-676.

(33) Kelley, S. O.; Barton, J. K. Science 1999, 283, 375.

(34) Wan, C.; Fiebig, T.; Kelley, S. O.; Treadway, C. R.; Barton, J. K.; Zewail, A. H. Proc. Natl. Acad. Sci. U.S.A. 1999, 96, 6014-6019.

(35) Rajski, S. R.; Kumar, S.; Roberts, R. J.; Barton, J. K. J. Am. Chem. Soc. 1999, 121,5615

(36) Yoshihara, K. Ultrafast Intermolecular Electron Transfer in Solution. In Electron Transfer: From Isolated Molecules to Biomolecules, 1st ed.; Jortner, J., Bixon, M., Eds.; John Wiley \& Sons: New York, 1999; Vol. 107, Part 2, pp 371-402.

(37) Kemnitz, K.; Yoshihara, K. Chem. Lett. 1991, 645-648.

(38) Kobayashi, T.; Takagi, Y.; Kandori, H.; Kemnitz, K.; Yoshihara, K. Chem. Phys. Lett. 1991, 180, 416-422.

(39) Kandori, H.; Kemnitz, K.; Yoshihara, K. J. Phys. Chem. 1992, 96, $8042-8048$

(40) Nagasawa, Y.; Yartsev, A. P.; Tominaga, K.; Johnson, A. E.; Yoshihara, K. J. Am. Chem. Soc. 1993, 115, 7922-7923.

(41) Yartsev, A.; Nagasawa, Y.; Douhal, A.; Yoshihara, K. Chem. Phys. Lett. 1993, 207, 546-550.

(42) Yoshihara, K.; Yartsev, A.; Nagasawa, Y.; Kandori, H.; Douhal, A.; Kemnitz, K. Pure Appl. Chem. 1993, 65, 1671-1675.

(43) Yoshihara, K.; Nagasawa, Y.; Yartsev, A. P.; Kumazaki, S.; Kandori, H.; Johnson, A. E.; Tominaga, K. J. Photochem. Photobiol. A. Chem. 1994, 80 .

(44) Nagasawa, Y.; Yartsev, A. P.; Tominaga, K.; Johnson, A. E.; Yoshihara, K. J. Chem. Phys. 1994, 101, 5717-5726.

(45) Nagasawa, Y.; Yartsev, A. P.; Tominaga, K.; Bisht, P. B.; Johnson, A. E.; Yoshihara, K. J. Phys. Chem. 1995, 99, 653-662.

(46) Pal, H.; Nagasawa, Y.; Tominaga, K.; Kumazaki, S.; Yoshihara, K. J. Chem. Phys. 1995, 102, 7758-7760

(47) Yoshihara, K.; Tominaga, K.; Nagasawa, Y. Bull. Chem. Soc. Jpn. 1995, 68, 696-712.
(48) Yoshihara, K. Dynamical Aspects of Intermolecular Electron Transfer. International Symposium on Electron Transfer in Proteins and Supramolecular Assemblies at Interfaces, 1996, Shonan Village, Kanagawa, Japan.

(49) Smith, N. A.; Lin, S.; Meech, S. R.; Shirota, H.; Yoshihara, K. J. Phys. Chem. A 1997, 101, 9578-9586.

(50) Shirota, H.; Pal, H.; Tominaga, K.; Yoshihara, K. J. Phys. Chem. A 1998, 102, 3089-3102.

(51) Shirota, H.; Pal, H.; Tominaga, K.; Yoshihara, K. Chem. Phys. 1998, 236, 355-364.

(52) Pal, H.; Shirota, H.; Tominaga, K.; Yoshihara, K. J. Chem. Phys. 1999, 110, 11454-11465.

(53) Rubtsov, I. V.; Shirota, H.; Yoshihara, K. J. Phys. Chem. A 1999, 103, 1801-1808

(54) Cave, R. J.; Newton, M. D. Chem. Phys. Lett. 1996, 249, 15-19.

(55) Cave, R. J.; Newton, M. D. J. Chem. Phys. 1997, 106, 9213 322

(56) Marcus, R. A.; Sutin, N. Biochim. Biophys. Acta 1985, 811, 265-

(57) Zusman, L. D. Chem. Phys. 1980, 49, 295-304.

(58) Zusman, L. D. Chem. Phys. 1983, 80, 29-43.

(59) Zusman, L. D. Russ. Chem. Phys. 1992, 61, 15-24.

(60) Pal, H.; Nagasawa, Y.; Tominaga, K.; Yoshihara, K. J. Phys. Chem. 1996, 100, 11964-11974.

(61) Walker, G. C.; Barbara, P. F.; Doorn, S. K.; Dong, Y.; Hupp, J. T. J. Phys. Chem. 1991, 95, 5712-5715.

(62) Åkesson, E.; Walker, G. C.; Barbara, P. F. J. Chem. Phys. 1991 $95,4188-4194$

(63) Tominaga, K.; Walker, G. C.; Jarzeba, W.; Barbara, P. F. J. Phys Chem. 1991, 95, 10475-10485.

(64) Barbara, P. F.; Walker, G. C.; Smith, T. P. Science 1992, 256, 975981.

(65) Walker, G. C.; Åkesson, E.; Johnson, A. E.; Levinger, N. E.; Barbara, P. F. J. Phys. Chem. 1992, 96, 3728-3736.

(66) Johnson, A. E.; Levinger, N. E.; Kliner, D. A. V.; Tominaga, K.; Barbara, P. F. Pure Appl. Chem. 1992, 64, 1219-1224.

(67) Tominaga, K.; Kliner, D. A. V.; Johnson, A. E.; Levinger, N. E.; Barbara, P. F. J. Chem. Phys. 1993, 98, 1228-1243.

(68) Sumi, H.; Marcus, R. A. J. Chem. Phys. 1986, 84, 4894-4914.

(69) Bixon, M.; Jortner, J. Chem. Phys. 1993, 176, 467-481.

(70) Jortner, J.; Bixon, M. J. Chem. Phys. 1988, 88, 167-170.

(71) Heitele, H. Angew. Chem., Int. Ed. Engl. 1993, 32, 359-377.

(72) Jones, G. I.; Griffin, S. F.; Choi, C.-y.; Bergmark, W. R. J. Org. Chem. 1984, 49, 2705-2708.

(73) Jones, G. I.; Jackson, W. R.; Choi, C.-y.; Bergmark, W. R. J. Phys. Chem. 1985, 89, 294-300.

(74) Horng, M. L.; Gardecki, J. A.; Papazyan, A.; Maroncelli, M. J. Phys. Chem. 1995, 99, 17311-17337.

(75) Jimenez, R.; Fleming, G. R.; Kumar, P. V.; Maroncelli, M. Nature 1994, 369, 471-473

(76) Barbara, P. F.; Jarzeba, W. Ultrafast photochemical intramolecular charge-transfer and excited-state solvation. In Advances in Photochemistry; Volman, D. H., Hammond, G. S., Gollnick, K., Eds.; John Wiley and Sons: NY, 1990; Vol. 15, pp 1-68.

(77) Shannon, C. F.; Eads, D. D. J. Chem. Phys. 1995, 103, 52085223

(78) Rosenthal, S. J.; Jimenez, R.; Fleming, G. R.; Kumar, P. V.; Maroncelli, M. J. Mol. Liq. 1994, 60, 25-56.

(79) Murakoshi, K.; Yanagida, S.; Capel, M.; Castner, E. W., Jr. Interfacial Electron-Transfer Dynamics of Photosensitized Zinc Oxide Nanoclusters. In Nanostructured Materials-Clusters, Thin Films, and Composites; Shalaev, V., Moskovits, M., Eds.; American Chemical Society: Washington, DC, 1997; Vol. 679, pp 221-238.

(80) English, D.; Das, K.; Ashby, K. D.; Park, J.; Petrich, J.; Castner, E. W., Jr. J. Am. Chem. Soc. 1997, 119, 11585-11590.

(81) National Instruments (Austin, TX) now sells and supports the nuLogic product line.

(82) Courtney, S. FITKL; New York, NY, 1996. 1997.

83) Cerius2, 3.5, 3.7 ed.; Molecular Simulations, Inc.: La Jolla, CA

(84) Mayo, S.; Olafson, B. D.; Goddard, W. A. I. J. Phys. Chem. 1990, 94, 8897.

(85) Brouwer, A. M.; Wilbrandt, R. J. Phys. Chem. 1996, 100, 96789688

(86) Rappe', A. K.; Goddard, W. A. I. J. Phys. Chem. 1991, 95, 3358.

(87) CRC Handbook of Physics and Chemistry, 65th ed.; Chemical Rubber Co.: Cleveland, OH, 1984.

(88) Hoover, W. Phys. Rev. 1985, A31, 1695.

(89) Verlet, L. Phys. Rev. 1967, 159, 98.

(90) Zerner, M. C. ZINDO, 3.7 ed.; Molecular Simulations, Inc.: San Diego, 1991.

(91) Coumarin. 337 is structurally similar to coumarin 343 but in C337 the 3-carboxylic acid group is replaced by a cyano group. 
(92) Wang, C.; Akhremitchev, B.; Walker, G. C. J. Phys. Chem. A 1997, $101,2735-2738$

(93) Fujita, E. Private communication.

(94) Chuang, T. J.; Cox, R. J.; Eisenthal, K. B. J. Am. Chem. Soc. 1974 $96,6828-6831$

(95) Seel, M.; Engleitner, S.; Zinth, W. Chem. Phys. Lett. 1997, 275, 363-369.

(96) Wolfseder, B.; Seidner, L.; Domcke, W.; Stock, G.; Seel, M.; Engleitner, S.; Zinth, W. Chem. Phys. 1998, 233, 323-334.

(97) Riddick, J. A.; Bunger, W. B.; Sakano, T. K. Organic Solvents. Physical Properties and Methods of Purification, 4th ed.; John Wiley and Sons: New York, 1986; Vol. 2.

(98) Onuchic, J. J. Chem. Phys. 1987, 86, 3925.

(99) Rips, I.; Jortner, J. J. Chem. Phys. 1987, 87, 2090.

(100) Newton, M. D. Chem. Rev. 1991, 91, 767-792.
(101) Henderson, T. M.; Cave, R. J. J. Chem. Phys. 1998, 109, 7414 (102) Miller, N. E.; Wander, M. C.; Cave, R. J. J. Phys. Chem. A 1999, 103, 1084-1093.

(103) Neyhart, G. A.; Timpson, C. J.; Bates, W. D.; Meyer, T. J. J. Am Chem. Soc. 1996, 118, 3730-3737.

(104) Stewart, J. J. P. J. Comput. Chem. 1989, 10, 221.

(105) Stewart, J. J. P. J. Comput. Chem. 1989, 10, 209-220.

(106) Stewart, J. J. P. J. Comput.-Aided Mol. Design 1990, 4, 1-105.

(107) Zinth, W.; Engleitner, S.; Seel, M. In Ultrafast Phenomena X;

Barbara, P. F., Fujimoto, J. G., Knox, W. H., Zinth, W., Eds.; Springer: Heidelberg, 1996; Vol. 62, pp 201-202.

(108) Engleitner, S.; Seel, M.; Zinth, W. J. Phys. Chem. 1999, 103 3013-3019.

(109) Xu, Q.-H.; Scholes, G. D.; Yang, M.; Fleming, G. R. J. Phys. Chem. A 1999, 103, 10348-10358. 